\title{
Estimates of the Green Function for the Fractional Laplacian Perturbed by Gradient
}

\author{
Krzysztof Bogdan • Tomasz Jakubowski
}

Received: 29 October 2010 / Accepted: 12 May 2011 / Published online: 7 June 2011

(C) The Author(s) 2011. This article is published with open access at Springerlink.com

\begin{abstract}
The Green function of the fractional Laplacian of the differential order bigger than one and the Green function of its gradient perturbations are comparable for bounded smooth multidimensional open sets if the drift function is in an appropriate Kato class.
\end{abstract}

Keywords Fractional Laplacian • Gradient perturbation • Green function • Smooth domain $\cdot$ Kato condition

Mathematics Subject Classifications (2010) 47 A55 • 60J35 • 60J50 • 60J75 • 47G20

\section{Introduction}

Perturbations of the Laplace operator $\Delta$ by the first order or gradient operators $b(x) \cdot \nabla$ were studied by Cranston and Zhao in [23]. They proved for Lipschitz domains that the Green function and the harmonic measure of $\Delta+b(x) \cdot \nabla$ are comparable with those of $\Delta$ under an appropriate Kato condition on the drift function $b$. Zhang then showed in [46] and [47] that the transition density of $\Delta+b \cdot \nabla$ has Gaussian bounds. The results were extended to more general second order elliptic operators by Liskevich and Zhang [40], and to drift measures satisfying the Kato condition by Kim and Song [36].

The fractional Laplacian $\Delta^{\alpha / 2}, 0<\alpha<2$, is a primary example of a non-local generator of a Markovian semigroup. Perturbations of $\Delta^{\alpha / 2}$ received much attention

The research was partially supported by MNiSW.

K. Bogdan · T. Jakubowski ( $\bowtie)$

Institute of Mathematics and Computer Science, Wrocław University of Technology,

Wybrzeże Wyspiańskiego 27, 50-370, Wrocław, Poland

e-mail: Tomasz.Jakubowski@pwr.wroc.pl

K. Bogdan

e-mail: Krzysztof.Bogdan@pwr.wroc.pl 
recently. In particular Schrödinger perturbations of $\Delta^{\alpha / 2}$ were studied by Chen and Song [19, 21], Bogdan and Byczkowski [7, 8], Bogdan et al. [6, 11]. Non-local Schrödinger-type perturbations were considered by Kim and Lee in [35], following earlier papers of Song [44, 45]. Gradient perturbations of $\Delta^{1 / 2}$ were studied by Caffarelli and Vasseur [16] and Kiselev et al. [37]. Gradient perturbations of $\Delta^{\alpha / 2}$ for $\alpha>1$ were considered by Bogdan and Jakubowski [12] and Jakubowski and Szczypkowski [34], with focus on sharp estimates of the corresponding transition densities on the whole of $\mathbb{R}^{d}$. In the present paper we estimate the Green function for smooth bounded subsets of $\mathbb{R}^{d}$.

Following [12] we let $\alpha \in(1,2)$. We will consider dimensions $d \in\{2,3, \ldots\}$, a nonempty bounded open $C^{1,1}$ set $D \subset \mathbb{R}^{d}$, its Green function $G_{D}$ for $\Delta^{\alpha / 2}$, and the Green function $\tilde{G}_{D}$ of the operator

$$
L=\Delta^{\alpha / 2}+b(x) \cdot \nabla
$$

where $b$ is a function in Kato class $\mathcal{K}_{d}^{\alpha-1}$ (for details see Section 2). Our interest in $L$ is motivated by the development of the classical theory of the Laplacian, nonsymmetry of $L$ (we have $L^{*}=\Delta^{\alpha / 2}-b(x) \cdot \nabla-\operatorname{div} b$ ), the fact that the drift is quite a problematic addition to a jump type process, and by a handful of techniques which already exist for $\Delta^{\alpha / 2}$.

The following estimate, aforementioned in the Abstract, is an extension to $\Delta^{\alpha / 2}$ of the results of Cranston and Zhao [23].

Theorem 1 Let $d \geq 2,1<\alpha<2, b \in \mathcal{K}_{d}^{\alpha-1}$, and let $D \subset \mathbb{R}^{d}$ be bounded and $C^{1,1}$. There exists a constant $C=C(\alpha, b, D)$ such that for $x, y \in D$,

$$
C^{-1} G_{D}(x, y) \leq \tilde{G}_{D}(x, y) \leq C G_{D}(x, y) .
$$

Sharp explicit estimates of $G_{D}$, hence of $\tilde{G}_{D}$, exist, see Eq. 24, and sharp explicit estimates of the corresponding Poisson kernel are given in Eq. 72 below. Theorem 1 is based on the perturbation formula for the Green operators,

$$
\tilde{G}_{D}=G_{D}+\tilde{G}_{D} b \nabla G_{D},
$$

where $b \nabla \varphi(x)=b(x) \cdot \nabla \varphi(x)$. Iterating yields formal perturbation series,

$$
\tilde{G}_{D}=\sum_{n=0}^{\infty} G_{D}\left(b \nabla G_{D}\right)^{n} .
$$

The structure of the proof of Theorem 1 is now as follows. Section 2 provides details on the $C^{1,1}$ condition and on transition densities, Green kernels and harmonic functions of the underlying Markov processes. In Section 3 we prove the perturbation formula and in Section 4 we prove that the perturbation series indeed converges and yields Eq. 1 for small sets $D$ with bounded distortion. In the proofs we use estimates for $G_{D}[20,30,38]$ and for the gradient of $G_{D}$ [14], the boundary Harnack inequality for $\Delta^{\alpha / 2}[5,13]$ and the Kato condition (30) for the drift function $b$. As a result in Section 4 we obtain the Harnack and boundary Harnack inequalities for nonnegative harmonic functions of $L$ in large open sets. These are then used in Section 5 along with the perturbation formula and a rough upper bound for $\tilde{G}_{D}$ given in Lemma 7, to 
prove Theorem 1 for arbitrary bounded $C^{1,1}$ open sets. A number of other auxiliary results are proved in the Appendix.

Concerning the statement of Theorem 1, we note that if the diameter of $D$ is smaller than $r$ and the distortion of $D$ is smaller than $\lambda$, then the constant $C$ in Eq. 1 depends only on $d, \alpha, r, \lambda$ and the suprema in the definition of the Kato class $\mathcal{K}_{d}^{\alpha}$ (see below).

We observe that an approach similar to ours was recently used for gradient perturbations of elliptic operators on small sets in [28] (see also [46]). In a wider perspective, Theorem 1 is an analogue of the Conditional Gauge Theorem (CGT) in the theory of Schrödinger perturbations, see [7, 8, 19, 21, 22] and [26]. We should remark here that the distributions of the Markov processes generated by $\Delta^{\alpha / 2}$ and $L$ are not mutually absolutely continuous locally in time even for (nonzero) constant drift $b$, and any $\alpha \in(0,2)$, see [42, Theorem 33.1]. Therefore techniques based on the Girsanov theorem [23] seem unavailable, and we need to proceed via analytic estimates of kernel functions. Apparently an adaptation of our arguments could be used to give a short analytic proof of CGT (compare [7, 19, 22]), in fact a proof much simpler than that of Theorem 1. Noteworthy, Green function estimates for Schrödinger perturbations hold conditionally under global assumptions of finiteness, e.g. gaugeability, existence of (finite) superharmonic functions bounded from below or smallness of the spectral radius. Lemma 7, a consequence of the estimates of the transition densities in [12], overrides such assumptions here. Heuristically, adding drift $b\left(X_{t}\right) d t$ to a stochastic process will not increase its mass on $\mathbb{R}^{d}$. This contrasts with a possibly exponential growth of the mass of Feynman-Kac semigroups generated by Schrödinger operators. The drift may, however, change the mass of the process killed off $D$ by trying to push it away from the fatal $D^{c}$. This is why Eq. 1 is nontrivial phenomenologically. Also the symmetry of the semigroup and Green function are lost in the presence of the drift, causing certain technical problems. In this connection we note that $\tilde{G}_{D}(y, x)$ may be considered the Green function of $L^{*}$, and this operator has non-zero Schrödinger part, namely - div $b$. Our results apply in particular to the Ornstein-Uhlenbeck operator $\Delta^{\alpha / 2}+k x \cdot \nabla$ (for dimensions $d \geq 2$ and $1<\alpha<2$ ). Here $k$ is a constant. We refer to [31] and [32] for estimates of superharmonic functions of this important operator. We note that for $k<0$ the drift function $b(x)=k x$ will generally increase the occupation time density (i.e. the Green function) for sets $D$ containing the origin.

The proof of Eq. 1 turned out to be quite difficult to handle, in terms of both the preliminaries and the auxiliary estimates of the Green function. Therefore we focused our attention on the more explicit $C^{1,1}$ open sets rather than Lipschitz open sets. We hope that our approach may now be adapted in the Lipschitz case. Here the sensitive elements are Lemma 9 and Eq. 47.

A few additional comments on possible extensions of the results are due. If $d=1<\alpha$, then the right hand side of Eq. 29 below will no longer be integrable. This explains our restriction to $d \geq 2$. We however conjecture that Theorem 1 does extend to $d=1$. This case is interesting even for the sake of the one-dimensional OrnsteinUhlenbeck process. One may wonder if Eq. 1 holds for $\alpha=1$, but we certainly know that Eq. 1 fails for $\alpha \in(0,1)$. Indeed, if $0<\alpha<1$ then the expected exit times from balls, to wit, $\int_{B\left(x_{0}, r\right)} G_{B\left(x_{0}, r\right)}(x, y) d y$, are generally incomparable for $\Delta^{\alpha / 2}$ and the Ornstein-Uhlenbeck operator $\Delta^{\alpha / 2}+k x \cdot \nabla$ (see [31]), so the Green functions are not comparable either. Heuristically, a (first-order) gradient perturbation is 
infinitesimally small with respect to $\Delta^{\alpha / 2}$ only if $\alpha>1$. This explains the restriction $1<\alpha<2$ in $[12,34]$ and the present paper. We remark that the existence of ratios and Martin representation of nonnegative harmonic functions of $L$ may likely be obtained with the results and toolbox presented in this paper and [13]. We also note that a similar approach should apply to additive perturbations of $\Delta^{\alpha / 2}$ by nonlocal Lévy-type operators (compare [24]), provided Eq. 29 can be generalized. It also seems possible and interesting to study drift perturbations of more general semigroups subordinated to the Gaussian semigroup [43].

\section{Preliminaries}

In what follows, $\mathbb{R}^{d}$ denotes the Euclidean space of dimension $d \geq 2, d y$ stands for the Lebesgue measure on $\mathbb{R}^{d}$, and we let

$$
1<\alpha<2 \text {. }
$$

Without further mention we will only consider Borelian sets, measures and functions in $\mathbb{R}^{d}$. By $x \cdot y$ we denote the Euclidean scalar product of $x, y \in \mathbb{R}^{d}$. We let $B(x, r)=$ $\left\{y \in \mathbb{R}^{d}:|x-y|<r\right\}$. For $D \subset \mathbb{R}^{d}$ we denote

$$
\delta_{D}(x)=\operatorname{dist}\left(x, D^{c}\right),
$$

the distance to the complement of $D$.

Definition 1 Nonempty open $D \subset \mathbb{R}^{d}$ is of class $C^{1,1}$ at scale $r>0$ if for every $Q \in$ д $D$ there are balls $B\left(x^{\prime}, r\right) \subset D$ and $B\left(x^{\prime \prime}, r\right) \subset D^{c}$ tangent at $Q$.

Thus, $B\left(x^{\prime}, r\right)$ and $B\left(x^{\prime \prime}, r\right)$ are the inner and outer balls tangent at $Q$, respectively. If $D$ is $C^{1,1}$ at some unspecified scale (hence also at all smaller scales), then we simply say $D$ is $C^{1,1}$. The localization radius,

$$
r_{0}=r_{0}(D)=\sup \left\{r: D \text { is } C^{1,1} \text { at scale } r\right\},
$$

refers to the local geometry of $D$, while the diameter,

$$
\operatorname{diam}(D)=\sup \{|x-y|: x, y \in D\},
$$

refers to the global geometry of $D$. The ratio $\operatorname{diam}(D) / r_{0}(D) \geq 2$ will be called the distortion of $D$. We can localize each $C^{1,1}$ open set as follows.

Lemma 1 There exists $\kappa>0$ such that if $D$ is $C^{1,1}$ at scale $r$ and $Q \in \partial D$, then there is $a C^{1,1}$ domain $F \subset D$ with $r_{0}(F)>\kappa r$, $\operatorname{diam}(F)<2 r$ and

$$
D \cap B(Q, r / 4)=F \cap B(Q, r / 4) .
$$

We will write $F=F(z, r)$, and we note that the distortion of $F$ is at most $2 / \kappa$, an absolute constant. The proof of Lemma 1 is given in the Appendix.

In what follows $D$ will be a nonempty bounded $C^{1,1}$ open set in $\mathbb{R}^{d}$.

We note that such $D$ may be disconnected but then it may only have a finite number of connected components, at a positive distance from each other. 
We will now give a brief review of the potential theory of the fractional Laplacian, and of the fractional Laplacian perturbed by gradient operators. The former case is well known $[2,7,9,13,39]$. The latter case is similar but we feel it calls for more details, and they are given in the Appendix.

Let $\mathcal{A}_{d, \gamma}=\Gamma((d-\gamma) / 2) /\left(2^{\gamma} \pi^{d / 2}|\Gamma(\gamma / 2)|\right)$ and

$$
v(y)=\mathcal{A}_{d,-\alpha}|y|^{-d-\alpha}, \quad y \in \mathbb{R}^{d} .
$$

The coefficient $\mathcal{A}_{d,-\alpha}$ is so chosen that

$$
\int_{\mathbb{R}^{d}}[1-\cos (\xi \cdot y)] v(y) d y=|\xi|^{\alpha}, \quad \xi \in \mathbb{R}^{d} .
$$

For (smooth compactly supported) $\phi \in C_{c}^{\infty}\left(\mathbb{R}^{d}\right)$, the fractional Laplacian is

$$
\Delta^{\alpha / 2} \phi(x)=\lim _{\varepsilon \downarrow 0} \int_{|y|>\varepsilon}[\phi(x+y)-\phi(x)] v(y) d y, \quad x \in \mathbb{R}^{d}
$$

(see [7,9] for a broader setup). If $x \notin \operatorname{supp} \phi$ then

$$
\Delta^{\alpha / 2} \phi(x)=\int_{\mathbb{R}^{d}} \phi(y) v(y-x) d y .
$$

If $r>0$ and $\phi_{r}(x)=\phi(r x)$ then

$$
\Delta^{\alpha / 2} \phi_{r}(x)=r^{\alpha} \Delta^{\alpha / 2} \phi(r x), \quad x \in \mathbb{R}^{d} .
$$

In this respect, $\Delta^{\alpha / 2}$ behaves like differentiation of order $\alpha$. We let $p_{t}$ be the smooth real-valued function on $\mathbb{R}^{d}$ with Fourier transform

$$
\int_{\mathbb{R}^{d}} p_{t}(x) e^{i x \cdot \xi} d x=e^{-t|\xi|^{\alpha}}, \quad t>0, \xi \in \mathbb{R}^{d} .
$$

According to Eq. 3 and the Lévy-Khinchine formula, $\left\{p_{t}\right\}$ is a probabilistic convolution semigroup with Lévy measure $v(y) d y$, see [42], [15] or [9]. Let

$$
p(t, x, y)=p_{t}(y-x) .
$$

Using Eq. 7 one proves that $p$ is the heat kernel of the fractional Laplacian:

$$
\int_{s}^{\infty} \int_{\mathbb{R}^{d}} p(u-s, x, z)\left[\partial_{u} \phi(u, z)+\Delta_{z}^{\alpha / 2} \phi(u, z)\right] d z d u=-\phi(s, x),
$$

where $s \in \mathbb{R}, x \in \mathbb{R}^{d}$ and $\phi \in C_{c}^{\infty}\left(\mathbb{R} \times \mathbb{R}^{d}\right)$.

We consider the time-homogeneous transition probability

$$
(t, x, A) \mapsto \int_{A} p(t, x, y) d y, \quad t>0, x \in \mathbb{R}^{d}, A \subset \mathbb{R}^{d} .
$$

By Kolmogorov's and Dinkin-Kinney's theorems the transition probability defines in the usual way Markov probability measures $\left\{\mathbb{P}^{x}, x \in \mathbb{R}^{d}\right\}$ on the space $\Omega$ of the right-continuous and left-limited functions $\omega:[0, \infty) \rightarrow \mathbb{R}^{d}$. We let $\mathbb{E}^{x}$ be the corresponding integrations. We will denote by $X=\left\{X_{t}\right\}_{t \geq 0}$ the canonical process on $\Omega, X_{t}(\omega)=\omega(t)$. In particular, according to Eq. 7,

$$
\mathbb{E}^{0} e^{i X_{t} \cdot \xi}=e^{-t|\xi|^{\alpha}}, \quad \xi \in \mathbb{R}^{d}, t \geq 0 .
$$


In fact, $\left(X, \mathbb{P}^{0}\right)$ is a Lévy process in $\mathbb{R}^{d}$ with zero Gaussian part and drift, and with $v(y) d y$ as the Lévy measure [42]. It follows from Eq. 7 that

$$
p_{t}(x)=t^{-d / \alpha} p_{1}\left(t^{-1 / \alpha} x\right), \quad t>0, x \in \mathbb{R}^{d} .
$$

It is well-known that $p_{1}(x) \stackrel{C}{\approx} 1 \wedge|x|^{-d-\alpha}$, hence

$$
p_{t}(x) \stackrel{C}{\approx} t^{-d / \alpha} \wedge \frac{t}{|x|^{d+\alpha}}, \quad t>0, x \in \mathbb{R}^{d} .
$$

Symbol $\stackrel{C}{\approx}$ means that either ratio of the sides is bounded by $C \in(0, \infty)$, and $C$ does not depend on the variables shown, here $t$ and $x$. We will write mere $\approx$ if $C$ is unimportant or understood. Constants will usually be denoted with generic $C$ (in statements) or $c$ (in proofs), and we will occasionally enumerate them for convenience of referencing. As usual, $a \wedge b=\min (a, b)$ and $a \vee b=\max (a, b)$. In what follows we will often use the identity

$$
a b=(a \wedge b)(a \vee b) .
$$

In view of Eq. 10 and the fact that each $p_{t}$ is a radial function, $X$ is called the isotropic $\alpha$-stable Lévy process (see $[15,42]$ for a discussion of general stable Lévy processes). We introduce the Riesz potential kernel (for $d>\alpha$ ),

$$
\mathcal{A}_{d, \alpha}|x|^{\alpha-d}=\int_{0}^{\infty} p_{t}(x) d t, \quad x \in \mathbb{R}^{d} .
$$

This is infinite if $x=0$, see Eq. 11 .

To study $\Delta^{\alpha / 2}$ with Dirichlet conditions we will consider the time of the first exit of the (canonical) process from $D$,

$$
\tau_{D}=\inf \left\{t>0: X_{t} \notin D\right\} .
$$

We let $\omega_{D}^{x}(B)=\mathbb{P}^{x}\left(X_{\tau_{D}} \in B\right)$, the $\alpha$-harmonic measure of $D[2,4,39]$. The joint distribution of $\left(\tau_{D}, X_{\tau_{D}}\right)$ defines the transition density of the process killed when leaving $D[3,22,27]$ :

$$
p_{D}(t, x, y)=p(t, x, y)-\mathbb{E}^{x}\left[\tau_{D}<t ; p\left(t-\tau_{D}, X_{\tau_{D}}, y\right)\right], \quad t>0, x, y \in \mathbb{R}^{d} .
$$

By Blumenthal's 0-1 law, radial symmetry of $p_{t}$ and $C^{1,1}$ geometry of the boundary of $\partial D$, we have $\mathbb{P}^{x}\left(\tau_{D}=0\right)=1$ for every $x \in D^{c}$. In particular, $p_{D}(t, x, y)=0$ if $x \in D^{c}$ or $y \in D^{c}$. By the strong Markov property,

$$
\mathbb{E}^{x}\left[t<\tau_{D} ; f\left(X_{t}\right)\right]=\int_{\mathbb{R}^{d}} f(y) p_{D}(t, x, y) d y, \quad t>0, x \in \mathbb{R}^{d},
$$

for functions $f \geq 0$. The Chapman-Kolmogorov equations hold for $p_{D}$,

$$
\int_{\mathbb{R}^{d}} p_{D}(s, x, z) p_{D}(t, z, y) d z=p_{D}(s+t, x, y), \quad s, t>0, x, y \in \mathbb{R}^{d} .
$$

Also, $p_{D}$ is jointly continuous when $t \neq 0$, and we have

$$
0 \leq p_{D}(t, x, y)=p_{D}(t, y, x) \leq p(t, x, y) .
$$


In particular,

$$
\int_{\mathbb{R}^{d}} p_{D}(t, x, y) d y \leq 1 .
$$

For $s \in \mathbb{R}, x \in \mathbb{R}^{d}$, and $\phi \in C_{c}^{\infty}(\mathbb{R} \times D)$, we have (compare Eq. 8)

$$
\int_{s}^{\infty} \int_{\mathbb{R}^{d}} p_{D}(u-s, x, z)\left[\partial_{u} \phi(u, z)+\Delta_{z}^{\alpha / 2} \phi(u, z)\right] d z d u=-\phi(s, x),
$$

which justifies calling $p_{D}$ the heat kernel of the (Dirichlet) fractional Laplacian on $D$. We define

$$
G_{D}(x, y)=\int_{0}^{\infty} p_{D}(t, x, y) d t, \quad x, y \in \mathbb{R}^{d} .
$$

It follows that $G_{D}(x, y)$ is symmetric and lower semi-continuous, and

$$
G_{D}(x, y)+\int_{D^{c}} \mathcal{A}_{d, \alpha}|y-z|^{\alpha-d} \omega_{D}^{x}(d z)=\mathcal{A}_{d, \alpha}|x-y|^{\alpha-d} .
$$

The Green operator of $\Delta^{\alpha / 2}$ for $D$ is

$$
G_{D} f(x)=\mathbb{E}^{x} \int_{0}^{\tau_{D}} f\left(X_{t}\right) d t=\int_{\mathbb{R}^{d}} G_{D}(x, y) f(y) d y, \quad x \in \mathbb{R}^{d},
$$

and we have

$$
G_{D}\left(\Delta^{\alpha / 2} \phi\right)(x)=-\phi(x), \quad x \in \mathbb{R}^{d}, \quad \phi \in C_{c}^{\infty}(D) .
$$

A result of Ikeda and Watanabe [29] asserts that for $x \in D$ the $\mathbb{P}^{x}$-distribution of $\left(\tau_{D}, X_{\tau_{D}-}, X_{\tau_{D}}\right)$ restricted to $X_{\tau_{D^{-}}} \neq X_{\tau_{D}}$ is given by the density function

$$
(s, u, z) \mapsto p_{D}(s, x, u) v(z-u) .
$$

The $C^{1,1}$ geometry of $D$ implies that $\mathbb{P}^{x}\left(X_{\tau_{D}-} \neq X_{\tau_{D}}\right)=1$ for $x \in D$ [5]. By Eqs. 17, 20 and Tonelli's theorem the $\mathbb{P}^{x}$-distribution of $X_{\tau_{D}}$ has a density function, called the Poisson kernel and defined as

$$
P_{D}(x, z)=\int_{D} G_{D}(x, y) v(z-y) d y .
$$

The Green function and Poisson kernel of the ball are known explicitly:

$$
\begin{gathered}
G_{B\left(x_{0}, r\right)}(x, v)=\mathcal{B}_{d, \alpha}|x-v|^{\alpha-d} \int_{0}^{w} \frac{s^{\alpha / 2-1}}{(s+1)^{d / 2}} d s, \\
P_{B\left(x_{0}, r\right)}(x, y)=\mathcal{C}_{d, \alpha}\left[\frac{r^{2}-\left|x-x_{0}\right|^{2}}{\left|y-x_{0}\right|^{2}-r^{2}}\right]^{\alpha / 2}|x-y|^{-d},
\end{gathered}
$$

where $\mathcal{B}_{d, \alpha}=\Gamma(d / 2) /\left(2^{\alpha} \pi^{d / 2}[\Gamma(\alpha / 2)]^{2}\right), \mathcal{C}_{d, \alpha}=\Gamma(d / 2) \pi^{-1-d / 2} \sin (\pi \alpha / 2)$,

$$
w=\left(r^{2}-\left|x-x_{0}\right|^{2}\right)\left(r^{2}-\left|v-x_{0}\right|^{2}\right) /|x-v|^{2},
$$

$\left|x-x_{0}\right|<r,\left|v-x_{0}\right|<r$, and $\left|y-x_{0}\right| \geq r$, see [4], [41] or [39]. 
The next estimate was proved by Kulczycki [38] and Chen and Song [20],

$$
\begin{aligned}
G_{D}(x, y) & \stackrel{C}{\approx}|x-y|^{\alpha-d}\left(\frac{\delta_{D}(x)^{\alpha / 2} \delta_{D}(y)^{\alpha / 2}}{|x-y|^{\alpha}} \wedge 1\right) \\
& \approx|x-y|^{\alpha-d} \frac{\delta_{D}(x)^{\alpha / 2} \delta_{D}(y)^{\alpha / 2}}{\left[\delta_{D}(x) \vee|x-y| \vee \delta_{D}(y)\right]^{\alpha}}, \quad x, y \in D .
\end{aligned}
$$

The reader may check equivalence of Eqs. 24 and 25 by first considering the case $\delta_{D}(x) \stackrel{3}{\approx} \delta_{D}(y)$. We like to remark that Eq. 25 may be also regarded a direct consequence of the approximate factorization of the Green function of Lipschitz open sets, see [30, Theorem 21]. It is well known that $C=C(d, \alpha, \lambda)$ in Eqs. 24 and 25 , if $\operatorname{diam}(D) / r_{0}(D) \leq \lambda$, i.e. $C$ may be so selected to depend only on $d, \alpha$ and (an upper bound for) the distortion of $D$. This follows from the proofs of [38] and [20] and is explicitly stated in [30].

We will consider a nonnegative function $u$ on $\mathbb{R}^{d}$, and an open set $U \subset \mathbb{R}^{d} \cdot u$ is called $\alpha$-harmonic on $U$ if for each open bounded $V \subset \bar{V} \subset U$,

$$
u(x)=\mathbb{E}^{x} u\left(X_{\tau_{V}}\right), \quad x \in V .
$$

We say that $u$ is regular $\alpha$-harmonic on $U$ if also

$$
u(x)=\mathbb{E}^{x} u\left(X_{\tau_{U}}\right), \quad x \in U .
$$

Here we assume absolute integrability of the expectations, and $\mathbb{E}^{x} u\left(X_{\tau_{U}}\right)$ is understood as $\mathbb{E}^{x}\left[\tau_{U}<\infty ; u\left(X_{\tau_{U}}\right)\right]$. For instance $x \mapsto G_{D}(x, y)$ is $\alpha$-harmonic in $D \backslash\{y\}$. In fact, by the strong Markov property, $G_{D}(x, y)=G_{V}(x, y)+\mathbb{E}^{x} G_{D}\left(X_{\tau_{V}}, y\right)$ for every open $V \subset U$, and $G_{V}(x, y)=0$ if $\operatorname{dist}(y, V)>0$ (see, e.g., [13]).

The following two results can be found in [5], see also [13].

Lemma 2 (Harnack Inequality) Let $x, y \in \mathbb{R}^{d}, s>0$ and $k \in \mathbb{N}$ satisfy $|x-y| \leq 2^{k}$. Let function $u$ be nonnegative in $\mathbb{R}^{d}$ and $\alpha$-harmonic in $B(x, s) \cup B(y, s)$. There is $C=C(d, \alpha)$ such that

$$
C^{-1} 2^{-k(d+\alpha)} u(x) \leq u(y) \leq C 2^{k(d+\alpha)} u(x) .
$$

Lemma 3 Let $Z \in \partial D$ and $r \in\left(0, r_{0}\right], 0<p<1$. Assume that functions $u, v$ are nonnegative in $\mathbb{R}^{d}$ and regular $\alpha$-harmonic and non-zero in $D \cap B\left(x_{0}, r\right)$. If $u$ and $v$ vanish on $D^{c} \cap B\left(x_{0}, r\right)$ then

$$
C^{-1} \frac{u(x)}{v(x)} \leq \frac{u(y)}{v(y)} \leq C \frac{u(x)}{v(x)},
$$

for $x, y \in D \cap B\left(x_{0}, p r\right)$. Here $C=C(d, \alpha, p)$.

We like to remark that the boundary Harnack inequality (Lemma 3) in fact holds for general open sets and is equivalent to an approximate factorization of the Poisson kernel of general open sets, see [13]. We encourage the reader to factorize $P_{B(0,1)}(x, y)$ when $x, y$ are not too close to each other. In passing we also note that an approximate factorization of $p_{D}(t, x, y)$ for Lipschitz domains is given in [10]. Concluding this part of our preliminary discussion we refer the reader to $[9,13]$ for more details and references. 
We note that $\alpha$-harmonic functions are smooth where $\alpha$-harmonic; use Eq. 23 or see [7]. The following gradient estimate is given in [14, Lemma 3.2].

Lemma 4 Let $U$ be an arbitrary open set in $\mathbb{R}^{d}$. For every nonnegative function $u$ on $\mathbb{R}^{d}$ which is $\alpha$-harmonic in $U$ we have

$$
|\nabla u(x)| \leq d \frac{u(x)}{\delta_{U}(x)}, \quad x \in U .
$$

Since $G_{U}(\cdot, y)$ is $\alpha$-harmonic in $U \backslash\{y\}$, for every $y \in U$ we obtain

$$
\left|\nabla_{x} G_{U}(x, y)\right| \leq d \frac{G_{U}(x, y)}{\delta_{U}(x) \wedge|x-y|}, \quad x, y \in U, x \neq y .
$$

We note in passing that a reverse inequality holds locally at the boundary of Lipschitz domains, with constant depending on the Lipschitz character of $D$ [14, Lemma 4.5]. In this sense Eqs. 28 and 29 are sharp. Also, $\nabla_{x} G_{U}(x, y)$ is jointly continuous for $x \neq y \in U$, see $[14,(10)]$.

Recall that $1<\alpha<2$. We say that vector field $b: \mathbb{R}^{d} \rightarrow \mathbb{R}^{d}$ belongs to the Kato class $\mathcal{K}_{d}^{\alpha-1}$ if

$$
\lim _{\varepsilon \rightarrow 0} \sup _{x \in \mathbb{R}^{d}} \int_{|x-z|<\varepsilon}|b(z)||x-z|^{\alpha-1-d} d z=0 .
$$

For instance, if $b$ is bounded or if $|b(z)| \leq|z|^{1-\alpha+\varepsilon}$ and $0<\varepsilon<\alpha-1$, then $b \in \mathcal{K}_{d}^{\alpha-1}$. Without much mention elements of $\mathcal{K}_{d}^{\alpha-1}$ will either be vector fields $\mathbb{R}^{d} \rightarrow \mathbb{R}^{d}$ or real-valued test functions $\mathbb{R}^{d} \rightarrow \mathbb{R}$, i.e. $\mathcal{K}_{d}^{\alpha-1}$ is more a condition than a class. Since $|x-z|^{\alpha-1-d}$ is locally bounded from below, $|b(z)| d z$ is a locally finite measure, and Eq. 30 is a local uniform integrability condition. If $b \in \mathcal{K}_{d}^{\alpha-1}$ and $f$ is bounded, then $f b \in \mathcal{K}_{d}^{\alpha-1}$, in particular, $f b$ is locally integrable. We note that $\mathcal{K}_{d}^{\alpha-1} \subset \mathcal{K}_{d}^{\alpha}$, where $\mathcal{K}_{d}^{\alpha}$ is defined by

$$
\lim _{\varepsilon \rightarrow 0} \sup _{x \in \mathbb{R}^{d}} \int_{|x-z|<\varepsilon}|b(z)||x-z|^{\alpha-d} d z=0 .
$$

Following [12] and [34] we recursively define, for $t>0$ and $x, y \in \mathbb{R}^{d}$,

$$
\begin{gathered}
p_{0}(t, x, y)=p(t, x, y), \\
p_{n}(t, x, y)=\int_{0}^{t} \int_{\mathbb{R}^{d}} p_{n-1}(t-s, x, z) b(z) \cdot \nabla_{z} p(s, z, y) d z d s, \quad n \geq 1,
\end{gathered}
$$

and we let

$$
\tilde{p}=\sum_{n=0}^{\infty} p_{n}
$$

The series converges absolutely, $\tilde{p}$ is a continuous probability transition density function, and

$$
c_{T}^{-1} p(t, x, y) \leq \tilde{p}(t, x, y) \leq c_{T} p(t, x, y), \quad x, y \in \mathbb{R}^{d}, 0<t<T,
$$

where $c_{T} \rightarrow 1$ if $T \rightarrow 0$, see [12, Theorem 2]. From a general perspective the approach of $[12,34]$ consist of using the semigroup as test functions, setting the 
assumptions on the perturbation so that $p_{1}$ is dominated by $p$ in short time, and recursively estimating multiple integrals defining $p_{n}$, so that the comparability with $p$ is preserved. Auxiliary estimates of $\nabla_{z} p(s, z, y)$ are obtained in $[12,34]$ by subordination to the Gaussian kernel, but the scope of the method is wider. For instance applications to Schrödinger perturbations of general transition densities are given in $[11,33]$.

We let $\tilde{\mathbb{P}}, \tilde{\mathbb{E}}$ be the Markov distributions and expectations defined by transition density $\tilde{p}$ on the canonical path space. We define the heat kernel of $L$ on $D$ by the usual G. Hunt's formula,

$$
\tilde{p}_{D}(t, x, y)=\tilde{p}(t, x, y)-\tilde{\mathbb{E}}^{x}\left[\tau_{D}<t ; \tilde{p}\left(t-\tau_{D}, X_{\tau_{D}}, y\right)\right] .
$$

We denote by $\tilde{G}_{D}(x, y)$ and $\tilde{G}_{D}$ the Green function and operator of $L$ on $D$,

$$
\begin{gathered}
\tilde{G}_{D}(x, y)=\int_{0}^{\infty} \tilde{p}_{D}(t, x, y) d t, \\
\tilde{G}_{D} \phi(x)=\int_{\mathbb{R}^{d}} \tilde{G}_{D}(x, y) \phi(y) d y .
\end{gathered}
$$

By Blumenthal's 0-1 law, $\tilde{p}_{D}(t, x, y)=0$ and $\tilde{G}_{D}(x, y)=0$ if $x \in D^{c}$ or $y \in D^{c}$, see Eq. 33. The next lemmas rely on the definition of $\tilde{p}$ and generalize results stated above for $\Delta^{\alpha / 2}$. The proofs of Lemmas 5, 6 and 8 are moved to the Appendix.

Lemma 5 For $s>0, x \in D$ and $\phi \in C_{c}^{\infty}((0, \infty) \times D)$ we have

$$
\int_{s}^{\infty} \int_{D} \tilde{p}_{D}(u-s, x, z)\left(\partial_{u}+\Delta_{z}^{\alpha / 2}+b(z) \cdot \nabla_{z}\right) \phi(u, z) d z d u=-\phi(s, x) .
$$

By Eq. 33 we have

$$
\lim _{t \rightarrow 0} \frac{\tilde{p}(t, x, y)}{t}=\lim _{t \rightarrow 0} \frac{p(t, x, y)}{t}=v(y-x) .
$$

Thus the intensity of jumps of the canonical process $X$ under $\tilde{\mathbb{P}}^{x}$ is the same as under $\mathbb{P}^{x}$. Accordingly, we obtain the following description.

Lemma 6 The $\tilde{\mathbb{P}}^{x}$-distribution of $\left(\tau_{D}, X_{\tau_{D}}\right)$ on $(0, \infty) \times(\bar{D})^{c}$ has density

$$
\int_{D} \tilde{p}_{D}(u, x, y) v(z-y) d y, \quad u>0, \delta_{D}(z)>0 .
$$

We define the Poisson kernel of $D$ for $L$,

$$
\tilde{P}_{D}(x, y)=\int_{D} \tilde{G}_{D}(x, z) v(y-z) d z, \quad x \in D, y \in D^{c} .
$$

By Eqs. 35, 38 and 37 we have

$$
\tilde{\mathbb{P}}^{x}\left(X_{\tau_{D}} \in A\right)=\int_{A} \int_{D} \tilde{G}_{D}(x, z) v(y-z) d z d y=\int_{A} \tilde{P}_{D}(x, y) d y,
$$

if $A \subset(\bar{D})^{c}$. For the case of $A \subset \partial D$, we refer the reader to Lemma 14 . 
The following rough estimate of $\tilde{G}_{D}$ results from the estimates of $\tilde{p}$ and the fact that $X$ jumps out of $D$ at least with intensity $\int_{|y|>\operatorname{diam}(D)} v(y) d y>0$.

Lemma $7 \tilde{G}_{D}(x, y)$ is continuous for $x \neq y, \tilde{G}_{D}(x, x)=\infty$ for $x \in D$, and

$$
\tilde{G}_{D}(x, y) \leq C_{0}|x-y|^{\alpha-d}, \quad x, y \in \mathbb{R}^{d},
$$

where $C_{0}=C_{0}(d, \alpha, \operatorname{diam}(D))$.

Proof We claim that there are constants $c$ and $C$ such that

$$
\tilde{p}_{D}(t, x, y) \leq C e^{-c t}, \quad t>1, \quad x, y \in \mathbb{R}^{d} .
$$

Indeed, let $\kappa_{D}(y)=\int_{D^{c}} v(z-y) d z$, so that $\kappa_{D}(y) \geq c>0$ for $y \in D$. Let $x \in D, t \geq 0$, and $F(t)=\tilde{\mathbb{P}}^{x}\left(\tau_{D}>t\right)=\int_{D} \tilde{p}_{D}(t, x, y) d y$. By Lemma 6 ,

$$
-F^{\prime}(t)=\int_{D} \tilde{p}_{D}(t, x, y) \kappa_{D}(y) d y \geq c F(t),
$$

hence $\tilde{\mathbb{P}}^{x}\left(\tau_{D}>t\right) \leq e^{-c t}$. By the semigroup property and Eq. 33, for $t>1$,

$$
\begin{aligned}
\tilde{p}_{D}(t, x, y) & \leq \int_{D} \tilde{p}_{D}(t-1, x, z) \tilde{p}(1, z, y) d z \\
& \leq c_{1} \int_{D} \tilde{p}_{D}(t-1, x, z) p(1, z, y) d z \\
& \leq c_{1} p(1,0,0) \tilde{\mathbb{P}}^{x}\left(\tau_{D}>t-1\right) \leq C e^{-c t} .
\end{aligned}
$$

By Eqs. 35, 33 and 40 we obtain

$$
\begin{aligned}
\tilde{G}_{D}(x, y) & \leq \int_{0}^{1} c_{1} p(t, x, y) d t+\int_{1}^{\infty} C e^{-c t} d t \\
& \leq \mathcal{A}_{d, \alpha}|x-y|^{\alpha-d}+C / c \leq\left(\mathcal{A}_{d, \alpha}+C \operatorname{diam}(D)^{d-\alpha} / c\right)|x-y|^{\alpha-d} .
\end{aligned}
$$

By Eq. 35 and dominated convergence theorem $\tilde{G}_{D}(x, y)$ is continuous if $x \neq y$, see Eqs. 33 and 11.

The next lemma results from integrating Eq. 36 against time.

Lemma 8 For all $\varphi \in C_{c}^{\infty}(D)$ and $x \in D$ we have

$$
\int_{D} \tilde{G}_{D}(x, z)\left(\Delta^{\alpha / 2} \varphi(z)+b(z) \cdot \nabla \varphi(z)\right) d z=-\varphi(x) .
$$

The definition of $L$-harmonicity is analogous to that of $\alpha$-harmonicity.

Definition $2 u$ is $L$-harmonic on $U$ if for each open bounded $V \subset \bar{V} \subset U$,

$$
u(x)=\tilde{\mathbb{E}}^{x} u\left(X_{\tau_{V}}\right), \quad x \in V .
$$

We say that $u$ is regular $L$-harmonic on $U$ if also

$$
u(x)=\tilde{\mathbb{E}}^{x} u\left(X_{\tau_{U}}\right), \quad x \in U .
$$


Here $\tilde{\mathbb{E}}^{x} u\left(X_{\tau_{U}}\right)=\tilde{\mathbb{E}}^{x}\left[\tau_{U}<\infty ; u\left(X_{\tau_{U}}\right)\right]$ and we always assume absolute integrability. In particular, $x \mapsto \tilde{G}_{D}(x, y)$ is $L$-harmonic in $D \backslash\{y\}$, in fact $\tilde{G}_{D}(x, y)=$ $\tilde{G}_{U}(x, y)+\tilde{\mathbb{E}}^{x} \tilde{G}_{V}\left(X\left(\tau_{U}\right), y\right)$ for every open $U \subset D$. We should note that in general $\tilde{G}_{D}(x, y) \neq \tilde{G}_{D}(y, x)$ (non-symmetry), and $y \mapsto \tilde{G}_{D}(x, y)$ is not $L$-harmonic. This accounts in part for the difficulties in estimating $\tilde{G}_{D}$.

\section{Perturbation Formula}

As before, $D$ is a bounded $C^{1,1}$ open set in $\mathbb{R}^{d}, b \in \mathcal{K}_{d}^{\alpha-1}$ and $1<\alpha<2 \leq d$. Let

$$
G=G_{D}, \tilde{G}=\tilde{G}_{D} \text { and } \delta=\delta_{D} .
$$

In view of Eq. 29 the next lemma yields uniform integrability of $b(z) \cdot \nabla_{z} G(y, z)$. In particular, the singularity $\delta(z)^{\alpha / 2-1}$ of $\nabla_{z} G$ at $\partial D$ integrates against $|b|$.

Lemma $9 G(y, z) /[\delta(z) \wedge|y-z|]$ is uniformly in y integrable against $|b(z)| d z$.

Proof In view of Eq. 25 it is enough to prove the uniform integrability of

$$
H(y, z)=|y-z|^{\alpha-d} \frac{\delta(y)^{\alpha / 2} \delta(z)^{\alpha / 2}}{[\delta(z) \vee|y-z| \vee \delta(y)]^{\alpha}} \frac{1}{\delta(z) \wedge|y-z|} .
$$

Let $A_{R}(y)=\{z \in D: H(y, z)>R\}$ for $R>0$. We will verify that

$$
\lim _{R \rightarrow \infty} \sup _{y \in D} \int_{A_{R}(y)} H(y, z)|b(z)| d z=0 .
$$

For $r>0$ we denote

$$
K_{r}=\sup _{x \in \mathbb{R}^{d}} \int_{B(x, r)}|b(y)||x-y|^{\alpha-1-d} d y .
$$

By Eq. 30 we have that $K_{r}<\infty$ and $K_{r} \downarrow 0$ as $r \downarrow 0$. For all $x \in \mathbb{R}^{d}$ and $r>0$,

$$
\int_{B(x, r)}|b(z)| d z \leq r^{d+1-\alpha} \int_{B(x, r)}|x-z|^{\alpha-1-d}|b(z)| d z \leq K_{r} r^{d+1-\alpha} .
$$

For $r>0$ we let $D(r)=\{z \in D: \delta(z)>r\}$. If $m>0, y \in D$ and $z \in D(\delta(y) / m)$, that is $\delta(z)>\delta(y) / m$, then we have

$$
|H(y, z)| \leq m^{1-\alpha / 2}|y-z|^{\alpha-1-d} .
$$

Indeed, by Eq. 12,

$$
\begin{aligned}
H(y, z) & =|y-z|^{\alpha-d} \frac{\delta(y)^{\alpha / 2} \delta(z)^{\alpha / 2}}{[\delta(z) \vee|y-z| \vee \delta(y)]^{\alpha}} \frac{\delta(z) \vee|y-z|}{\delta(z)|y-z|} \\
& \leq|y-z|^{\alpha-1-d} \delta(z)^{\alpha / 2-1} \delta(y)^{\alpha / 2}[\delta(z) \vee|y-z| \vee \delta(y)]^{1-\alpha} \\
& \leq|y-z|^{\alpha-1-d} \delta(z)^{\alpha / 2-1} \delta(y)^{1-\alpha / 2} \leq m^{1-\alpha / 2}|y-z|^{\alpha-1-d} .
\end{aligned}
$$


If $R \rightarrow \infty$, then uniformly in $y$ we have

$$
\begin{aligned}
& \int_{D(\delta(y) / m) \cap A_{R}(y)} H(y, z)|b(z)| d z \\
& \quad \leq m^{1-\alpha / 2} \int_{\left\{z \in D:|y-z|^{\alpha-1-d}>R m^{\alpha / 2-1}\right\}}|y-z|^{\alpha-1-d}|b(z)| d z \rightarrow 0 .
\end{aligned}
$$

For $y \in D, k, n \geq 0$ and $m \geq 2$ we consider

$$
W_{n, k}^{m}(y)=\left\{z \in D: \frac{\delta(y)}{m 2^{n+1}}<\delta(z) \leq \frac{\delta(y)}{m 2^{n}}, k \delta(y)<|y-z| \leq(k+1) \delta(y)\right\} .
$$

$W_{n, k}^{m}(y)$ may be covered by $c_{1}(k+1)^{d-2} m^{d-1} 2^{n(d-1)}$ balls of radii $\frac{\delta(y)}{m 2^{n}}$, thus

$$
\begin{aligned}
\int_{W_{n, k}^{m}(y)}|b(z)| d z & \leq c_{1}(k+1)^{d-2} m^{d-1} 2^{n(d-1)} \sup _{x \in \mathbb{R}^{d}} \int_{B\left(x, \delta(y) / m 2^{n}\right)}|b(z)| d z \\
& \leq c_{1} K_{\delta(y) / m 2^{n}}(k+1)^{d-2} m^{d-1} 2^{n(d-1)}\left(\frac{\delta(y)}{m 2^{n}}\right)^{d+1-\alpha} \\
& =c_{1} K_{\delta(y) / m 2^{n}}(k+1)^{d-2} m^{\alpha-2} 2^{n(\alpha-2)} \delta(y)^{d+1-\alpha} .
\end{aligned}
$$

For $z \in W_{n, k}^{m}(y)$ we have $\delta(y) \geq 2 \delta(z)$, hence $|y-z| \geq \delta(y) / 2$ and $|y-z| \geq \delta(z)$. We obtain

$$
\begin{aligned}
\int_{A_{R}(y) \backslash D(\delta(y) / m)} & H(y, z)|b(z)| d z \\
& \leq \sum_{n=0}^{\infty} \sum_{k=0}^{\infty} \int_{W_{n, k}^{m}(y)} \frac{\delta(y)^{\alpha / 2}}{|y-z|^{d} \delta(z)^{1-\alpha / 2}}|b(z)| d z \\
& \leq \sum_{n=0}^{\infty} \sum_{k=0}^{\infty} \int_{W_{n, k}^{m}(y)} \frac{\delta(y)^{\alpha / 2}}{((k+1) \delta(y) / 2)^{d}\left[\delta(y) /\left(m 2^{n+1}\right)\right]^{1-\alpha / 2}}|b(z)| d z \\
& \leq c_{2} K_{\delta(y) / m} \sum_{n=0}^{\infty} \sum_{k=0}^{\infty}(k+1)^{d-2} m^{\alpha-2} 2^{n(\alpha-2)} 2^{d}(k+1)^{-d} 2^{n(1-\alpha / 2)} m^{1-\alpha / 2} \\
& \leq c_{3} m^{\alpha / 2-1} K_{\delta(y) / m} .
\end{aligned}
$$

Let $\varepsilon>0$. We chose $m$ and $R$ so large that $c_{3} m^{\alpha / 2-1} K_{\operatorname{diam}(D) / m}<\varepsilon / 2$ and

$$
\sup _{y \in D} \int_{D(\delta(y) / m) \cap A_{R}(y)} H(y, z)|b(z)| d z<\varepsilon / 2 .
$$

This completes the proof.

We consider the operator $b \nabla$ :

$$
(b \nabla \phi)(x)=b(x) \cdot \nabla \phi(x)=\sum_{i=1}^{d} b_{i}(x) \frac{\partial \phi(x)}{\partial x_{i}} .
$$


We will study the perturbation series $\sum_{n=0}^{\infty}(G b \nabla)^{n} G$ of integral operators on $\mathcal{K}_{d}^{\alpha-1}$. Namely we will apply $G b \nabla G$ to real-valued $f \in \mathcal{K}_{d}^{\alpha-1}$ :

$$
G b \nabla G f(x)=\int_{D} G(x, z) b(z) \cdot \nabla_{z} \int_{D} G(z, y) f(y) d y d z .
$$

We will need to interchange the integration and differentiation in Eq. 43.

Lemma 10 Let $1<\alpha<2$. If $f \in \mathcal{K}_{d}^{\alpha-1}$ or at least $G(z, y) /|y-z|$ is locally in $z \in D$ uniformly integrable against $|f(y)| d y$, then

$$
\nabla_{z} \int_{D} G(z, y) f(y) d y=\int_{D} \nabla_{z} G(z, y) f(y) d y, \quad z \in D .
$$

Proof The result is proved for $f \in \mathcal{K}_{d}^{\alpha-1}$ in [14, Lemma 5.2]. For the more general $f$ we note that $G(z, y)$ and $\nabla_{z} G(z, y)$ are continuous on $D \times D$ except at $z=$ $y$, see the remark following Eq. 29. They are also uniformly integrable against $|f(y)| d y$ for $z$ in compact subsets of $D$. In consequence $g(z)=G f(z)$ and $k(z)=$ $\int_{D} \nabla_{z} G(z, y) f(y) d y$ are continuous on $D$. We consider $f_{n}=f \wedge n \vee(-n) \in \mathcal{K}_{d}^{\alpha-1}$, $g_{n}=G f_{n}$. We have $g_{n} \rightarrow g$ and $\nabla g_{n} \rightarrow k$. It follows that $\nabla g=k$.

For $x, y \in D$ we let

$$
\begin{aligned}
& \kappa(x, y)=\int_{D}|b(z)| \frac{G(x, z) G(z, y)}{G(x, y)(\delta(z) \wedge|y-z|)} d z, \\
& \hat{\kappa}(x, y)=\int_{D}|b(z)| \frac{G(x, z) G(z, y)(\delta(x) \wedge|x-y|)}{G(x, y)(\delta(z) \wedge|y-z|)(\delta(x) \wedge|x-z|)} d z .
\end{aligned}
$$

In what follows $\kappa$ and $\hat{\kappa}$ will serve as majorants for the perturbation series.

Lemma 11 Let $\lambda, r<\infty$. There is $C_{1}=C_{1}(d, \alpha, b, \lambda, r)$ such that if $D$ is $C^{1,1}$, $\operatorname{diam}(D) / r_{0}(D) \leq \lambda$ and $\operatorname{diam}(D) \leq r$, then $\kappa(x, y) \leq C_{1}, \hat{\kappa}(x, y) \leq 2 C_{1}$ for $x, y \in D$, and $C_{1}(d, \alpha, b, \lambda, r) \rightarrow 0$ as $r \rightarrow 0$.

Proof Denote $g(x)=\delta(x)^{\alpha / 2}$. Let $x, z \in D$. By Eq. 25 we have

$$
\begin{aligned}
\frac{g(z)}{g(x)} G(x, z) & \approx \frac{g^{2}(z)}{(\delta(x) \vee|x-z| \vee \delta(z))^{\alpha}}|x-z|^{\alpha-d} \\
& \leq\left(\frac{\delta(z)}{\delta(z) \vee|x-z|}\right)^{\alpha}|x-z|^{\alpha-d} \leq \frac{\delta(z)}{\delta(z) \vee|x-z|}|x-z|^{\alpha-d} \\
& =(\delta(z) \wedge|x-z|)|x-z|^{\alpha-1-d} .
\end{aligned}
$$

Let $\mathcal{G}(x, y)=G(x, y) /[g(x) g(y)]$. The so-called 3G Theorem holds for $\mathcal{G}$ :

$$
\mathcal{G}(x, z) \wedge \mathcal{G}(z, y) \leq c \mathcal{G}(x, y), \quad x, y, z \in D,
$$


where $c$ depends only on $d, \alpha$ and the distortion of $D$ [25]. We obtain

$$
\begin{aligned}
\frac{G(x, z) G(z, y)}{G(x, y)} & =g^{2}(z) \frac{\mathcal{G}(x, z) \mathcal{G}(z, y)}{\mathcal{G}(x, y)} \leq c\left(\frac{g(z)}{g(x)} G(x, z) \vee \frac{g(z)}{g(y)} G(z, y)\right) \\
& \leq c\left(\frac{\delta(z) \wedge|x-z|}{|x-z|^{d+1-\alpha}} \vee \frac{\delta(z) \wedge|y-z|}{|y-z|^{d+1-\alpha}}\right) \\
& =c(\delta(z) \wedge|x-z| \wedge|y-z|)\left(\frac{1}{|x-z|^{d+1-\alpha}} \vee \frac{1}{|y-z|^{d+1-\alpha}}\right) .
\end{aligned}
$$

We have

$$
\frac{G(x, z) G(z, y)}{G(x, y)(\delta(z) \wedge|y-z| \wedge|x-z|)} \leq c\left(|x-z|^{\alpha-1-d}+|y-z|^{\alpha-1-d}\right),
$$

so we actually have uniform integrability against $|b(z)| d z$. The statement about $\kappa$ follows form (30).

To estimate $\hat{\kappa}$ we consider two cases. If $2|x-z|>\delta(x) \wedge|x-y|$, then

$$
\frac{\delta(z) \wedge|x-z| \wedge|y-z|}{(\delta(z) \wedge|y-z|)(\delta(x) \wedge|x-z|)} \leq \frac{1}{\delta(x) \wedge|x-z|} \leq \frac{2}{\delta(x) \wedge|x-y|} .
$$

If $2|x-z| \leq \delta(x) \wedge|x-y|$, then $\delta(z) \geq \delta(x) / 2,|y-z| \geq|x-y| / 2$, and so

$$
\frac{\delta(z) \wedge|x-z| \wedge|y-z|}{(\delta(z) \wedge|y-z|)(\delta(x) \wedge|x-z|)} \leq \frac{\delta(z) \wedge|x-z| \wedge|y-z|}{(\delta(x) / 2 \wedge|x-y| / 2)|x-z|} \leq \frac{2}{\delta(x) \wedge|x-y|} .
$$

By Eqs. 49 and 30 we obtain $\hat{\kappa}(x, y) \leq 2 C_{1}$. In fact we observe the uniform integrability against $|b(z)| d z$. The above estimates of the factors in Eqs. 45 and 46 depend on $D$ only through $d$ and $\operatorname{diam}(D) / r_{0}(D)$ [30]. Therefore the integrals in Eqs. 45 and 46 are arbitrarily small if $\operatorname{diam}(D)$ is small enough, and the distortion of $D$ is bounded by a constant. This follows from Eq. 30. If $\operatorname{diam}(D)$ is not small but finite then we only have the boundedness of $\kappa$ and $\hat{\kappa}$, which also follows from Eq. 30.

For $x \neq y$ we let

$$
G_{1}(x, y)=\int_{D} G(x, z) b(z) \cdot \nabla_{z} G(z, y) d z
$$

By Eqs. 29 and 45, and Lemma 11 the integral is absolutely convergent,

$$
\left|G_{1}(x, y)\right| \leq d G(x, y) \int_{D} \frac{|b(z)| G(x, z) G(z, y)}{G(x, y)(\delta(z) \wedge|y-z|)} d z \leq d C_{1} G(x, y) .
$$

For $f \in \mathcal{K}_{d}^{\alpha-1} \subset \mathcal{K}_{d}^{\alpha}$ we have

$$
\begin{aligned}
& \int_{D} G(x, y) \int_{D}|b(z)| \frac{G(x, z) G(z, y)}{G(x, y)(\delta(z) \wedge|y-z|)} d z|f(y)| d y \\
& \quad \leq C_{1} \int_{D} G(x, y)|f(y)| d y<\infty,
\end{aligned}
$$


hence by Lemma 10, Eq. 29 and Fubini's theorem,

$$
\begin{aligned}
G b \nabla G f(x) & =\int_{D} G(x, z) \int_{D} b(z) \cdot \nabla_{z} G(z, y) f(y) d y d z \\
& =\int_{D} G_{1}(x, y) f(y) d y .
\end{aligned}
$$

We like to note that the linear map $f \mapsto b \nabla G f$ preserves $\mathcal{K}_{d}^{\alpha-1}$ because $\nabla G f$ is a bounded function, see Lemma 9 and the remarks following Eq. 30.

We will now prove the pointwise perturbation formula.

Lemma 12 Let $x, y \in \mathbb{R}^{d}, x \neq y$. We have

$$
\tilde{G}(x, y)=G(x, y)+\int_{D} \tilde{G}(x, z) b(z) \cdot \nabla_{z} G(z, y) d z .
$$

Proof Let $x \in D$. For $\phi \in \mathcal{K}_{d}^{\alpha-1}$ we consider

$$
\Lambda(\phi)=\int_{\mathbb{R}^{d}}\left[\tilde{G}(x, y)-G(x, y)-\int_{\mathbb{R}^{d}} \tilde{G}(x, z) b(z) \cdot \nabla_{z} G(z, y) d z\right] \phi(y) d y .
$$

By Lemmas 9 and 7, the iterated integral converges absolutely. If $\varphi \in C_{c}^{\infty}(D)$ and $\phi=\Delta^{\alpha / 2} \varphi$, then using Eqs. 41 and 19, and Lemma 10 we obtain

$$
\Lambda(\phi)=-\varphi(x)-\tilde{G}(b \nabla \varphi)(x)+\varphi(x)-\tilde{G}(b \nabla(-\varphi))(x)=0 .
$$

By [7, Theorem 3.12], $\Lambda(\phi)=\int_{\mathbb{R}^{d}} \phi(y) \lambda(y) d y$, where $\lambda$ is $\alpha$-harmonic on $D$. Since our $\lambda$ is bounded near $\partial D$ and vanishes on $D^{c}$, we have that $\lambda \equiv 0$, see [7, Lemma 17]. By uniform integrability and the remark following Eq. 29 and by Lemma 7 we see that both sides of Eq. 52 are continuous in $y \in D \backslash\{x\}$, hence we have pointwise equality in Eq. 52.

In addition to $G_{1}$ we inductively define

$$
G_{n}(x, y)=\int G_{n-1}(x, z) b(z) \cdot \nabla_{z} G(z, y) d z, \quad x \neq y \in D, \quad n=2,3, \ldots
$$

We also let $G_{0}(x, y)=G(x, y)$. By Eqs. 29, 51 and 45, and Lemma 11 and induction,

$$
\begin{aligned}
\left|G_{n}(x, y)\right| & \leq \int_{D}\left|G_{n-1}(x, z)\right||b(z)|\left|\nabla_{z} G(z, y)\right| d z \\
& \leq\left(C_{1} d\right)^{n-1} \int_{D}|b(z)| G(x, z)\left|\nabla_{z} G(z, y)\right| d z \leq\left(C_{1} d\right)^{n} G(x, y),
\end{aligned}
$$

where $n=0,1,2, \ldots$ and $x \neq y$. By Eq. 53 and induction we prove that

$$
\int_{D} G_{n}(x, z) \int_{D} b(z) \cdot \nabla_{z} G(z, y) f(y) d y d z=\int_{D} G_{n+1}(x, y) f(y) d y,
$$

hence $G_{n}$ is the integral kernel of $G(b \nabla G)^{n}$,

$$
G(b \nabla G)^{n} f(x)=\int_{D} G_{n}(x, y) f(y) d y, \quad f \in \mathcal{K}_{d}^{\alpha-1}, \quad x \in D .
$$


We can also handle the gradient of $G_{n}$. Namely, for $x, y \in D, x \neq y$, and $n=1,2, \ldots$, we have:

$$
\begin{aligned}
& \left|\nabla_{x} G_{n-1}(x, y)\right| \leq\left(2 C_{1}\right)^{n-1} d^{n} \frac{G(x, y)}{\delta(x) \wedge|x-y|}, \\
& G_{n}(x, y)=\int G(x, z) b(z) \cdot \nabla_{z} G_{n-1}(z, y) d z,
\end{aligned}
$$

and

$$
\nabla_{x} G_{n}(x, y)=\int \nabla_{x} G_{n-1}(x, z) b(z) \cdot \nabla_{z} G(z, y) d z .
$$

The inequality and the equalities are proved consecutively by induction. In the process we use Lemma 10, Eq. 46, estimates following Eq. 49 in the proof of Lemma 11, and Fubini's theorem.

Iterating Eq. 52, by Eq. 55 we obtain for $n=0,1, \ldots$, and $x \neq y$,

$$
\begin{aligned}
\tilde{G}(x, y) & =G(x, y)+\int \tilde{G}(x, z) b(z) \cdot \nabla_{z} G(z, y) d z \\
& =\sum_{k=0}^{n} G_{k}(x, y)+\int \tilde{G}(x, z) b(z) \cdot \nabla_{z} G_{n}(z, y) d z .
\end{aligned}
$$

The details are left to the reader.

\section{Local Results}

We will prove and use the comparability of $G_{S}$ and $\tilde{G}_{S}$ for small smooth sets, $S$, of class $C^{1,1}$ (this part of our development is similar to [28]). The following is a variant of Khasminski's lemma [22].

Lemma 13 Let $d \geq 2,1<\alpha<2, b \in \mathcal{K}_{d}^{\alpha-1}$ and $\lambda>0$. There is $\varepsilon=\varepsilon(d, \alpha, b, \lambda)>0$ such that if $\operatorname{diam}(S) / r_{0}(S) \leq \lambda$ and $\operatorname{diam}(S) \leq \varepsilon$, then

$$
\frac{2}{3} G_{S}(x, y) \leq \tilde{G}_{S}(x, y) \leq \frac{4}{3} G_{S}(x, y), \quad x, y \in \mathbb{R}^{d} .
$$

Proof Let $D=S$. By Lemma 11 and Eq. 53 there is $\varepsilon=\varepsilon(d, \alpha, b, \lambda)>0$ and

$$
\left|G_{n}(x, y)\right| \leq 4^{-n} G(x, y), \quad x \neq y, n=0,1, \ldots,
$$

provided $\operatorname{diam}(D) / r_{0}(D) \leq \lambda$ and $r_{0}(D) \leq \varepsilon$. For $x \neq y$ we have $\tilde{G}(x, y)=$ $\sum_{n=0}^{\infty} G_{n}(x, y)$. Indeed, the remainder in Eq. 57 is bounded by

$$
c \int_{D}|x-z|^{\alpha-d}|b(z)|\left(2 C_{1}\right)^{n-1} d^{n} \frac{G(z, y)}{\delta(z) \wedge|y-z|} d z \rightarrow 0, \quad \text { as } n \rightarrow \infty .
$$

Here $2 C_{1} d \leq 1 / 2$ and the integral is finite because of Lemma 9 and Eq. 31. Thus,

$$
\tilde{G}(x, y)=\sum_{n=0}^{\infty} G_{n}(x, y) \leq \sum_{n=0}^{\infty} 4^{-n} G(x, y)=\frac{4}{3} G(x, y),
$$


and

$$
\tilde{G}(x, y) \geq G(x, y)-\sum_{n=1}^{\infty} 4^{-n} G(x, y)=\frac{2}{3} G(x, y) .
$$

We like to note that the comparison constants in the above proof will improve to 1 if $\operatorname{diam}(S) \rightarrow 0$ and the distortion of $S$ is bounded. By Eq. 39,

$$
\tilde{\mathbb{P}}^{x}\left(X_{\tau_{S}} \in A\right) \approx \mathbb{P}^{x}\left(X_{\tau_{S}} \in A\right), \quad x \in S, \quad A \subset(\bar{S})^{c} .
$$

We are in a position to prove that the boundary of our general $C^{1,1}$ open set $D$ is not hit at the first exit (recall that $1<\alpha<2$ ).

Lemma 14 For every $x \in D$ we have that $\tilde{\mathbb{P}}^{x}\left(X_{\tau_{D}} \in \partial D\right)=0$.

Proof Let $u(x)=\tilde{\mathbb{P}}^{x}\left(X_{\tau_{D}} \in \partial D\right), x \in \mathbb{R}^{d}$. We claim that there exists $c=c(d, \alpha, D, b)$ $>0$ such that $u(x)<1-c$ for $x \in D$. Indeed, we consider small $\varepsilon>0, x \in D$, $r=\varepsilon \operatorname{dist}\left(x, D^{c}\right)$, the ball $B=B(x, r / 2) \subset D$, and a ball $B^{\prime} \subset(\bar{D})^{c}$ with radius and distance to $B$ comparable with $r$. By Eqs. 59 and 23,

$$
\tilde{\mathbb{P}}^{x}\left(X_{\tau_{D}} \notin \partial D\right) \geq \tilde{\mathbb{P}}^{x}\left(X_{\tau_{B}(x, r / 2)} \in B^{\prime}\right) \approx \mathbb{P}^{x}\left(X_{\tau_{B}(x, r / 2)} \in B^{\prime}\right) \geq c .
$$

Furthermore, let $D_{n}=\left\{y \in D: \operatorname{dist}\left(y, D^{c}\right)>1 / n\right\}, n=1,2, \ldots$ We consider $n$ such that $B(x, r / 2) \subset D_{n}$. We have $\tilde{\mathbb{P}}^{x}\left(X_{\tau_{D_{n}}} \in \bar{D}\right) \leq 1-\tilde{\mathbb{P}}^{x}\left(X_{\tau_{B}} \in B^{\prime}\right) \leq 1-c$, as before. Let $C=\sup \{u(y): y \in D\}$. We have $u(x)=\tilde{\mathbb{E}}^{x}\left\{u\left(X_{\tau_{D_{n}}}\right) ; X_{\tau_{D_{n}}} \in \bar{D}\right\} \leq C(1-$ $c)$, hence $C \leq C(1-c)$ and so $C=0$.

In the context of Lemma 13 , the $\tilde{\mathbb{P}}^{x}$ distribution of $X_{\tau_{S}}$ is absolutely continuous with respect to the Lebesgue measure, and has density function

$$
\tilde{P}_{S}(x, y) \approx P_{S}(x, y), \quad y \in S^{c},
$$

provided $x \in S$. This follows from Eq. 39 and Lemma 14. For clarity,

$$
\tilde{\mathbb{P}}^{x}\left(X_{\tau_{S}} \in A\right) \approx \mathbb{P}^{x}\left(X_{\tau_{S}} \in A\right), \quad x \in S, \quad A \subset S^{c} .
$$

Lemma 15 (Harnack Inequality for $L$ ) Let $x, y \in \mathbb{R}^{d}, 0<s<1$ and $k \in \mathbb{N}$ satisfy $\mid x-$ $y \mid \leq 2^{k}$ s. Let $\tilde{u}$ be nonnegative in $\mathbb{R}^{d}$ and L-harmonic in $B(x, s) \cup B(y, s)$. There is $C=C(d, \alpha, b)$ such that

$$
C^{-1} 2^{-k(d+\alpha)} \tilde{u}(x) \leq \tilde{u}(y) \leq C 2^{k(d+\alpha)} \tilde{u}(x) .
$$

Proof We may assume that $s \leq 1 \wedge \varepsilon / 2$, with $\varepsilon$ of Lemma 13. Let $u(z)=\tilde{u}(z)$ for $z \in B(y, 2 s / 3)^{c}$ and $u(z)=\int_{B(y, 2 s / 3)^{c}} \tilde{u}(v) P_{B(y, 2 s / 3)}(z, v) d v$ for $z \in B(y, 2 s / 3)$, so that $u$ is nonnegative in $\mathbb{R}^{d}$ and (regular) $\alpha$-harmonic in $B(y, 2 s / 3)$. Let $z \in B(y, s / 2)$. By Eq. 61,

$$
\tilde{u}(z)=\tilde{\mathbb{E}}^{z} \tilde{u}\left(X\left(\tau_{B(y, 2 s / 3)}\right)\right)=\int_{B(y, 2 s / 3)^{c}} \tilde{u}(v) \tilde{P}_{B(y, 2 s / 3)}(z, v) d v \approx u(z) .
$$


By Lemma 2 we get $\tilde{u}(z) \approx \tilde{u}(y)$ with a constant depending only on $d$, $\alpha$ and $b$. To compare $\tilde{u}(x)$ and $\tilde{u}(y)$ we will assume that $|x-y| \geq 3 s / 2$, because otherwise we may take smaller $s$. For $z \in B(y, s / 2)$ we have $|x-z| \leq|x-y|+|y-z| \leq 2^{k} s+s / 2 \leq$ $2^{k+1} s$, and we get

$$
\begin{aligned}
P_{B(x, s / 2)}(x, z) & =C_{d, \alpha}\left(\frac{(s / 2)^{2}}{|x-z|^{2}-(s / 2)^{2}}\right)^{\alpha / 2} \frac{1}{|x-z|^{d}} \\
& \geq C_{d, \alpha} 2^{-\alpha} s^{\alpha}|x-z|^{-d-\alpha} \\
& \geq C_{d, \alpha} 2^{-(d+2 \alpha)} 2^{-k(d+\alpha)} s^{-d} .
\end{aligned}
$$

Since $\tilde{P}_{B(x, s / 2)} \approx P_{B(x, s / 2)}$, by the first part of the proof we obtain

$$
\begin{aligned}
\tilde{u}(x) & =\int_{B(x, s / 2)^{c}} \tilde{P}_{B(x, s / 2)}(x, z) \tilde{u}(z) d z \geq \int_{B(y, s / 2)} \tilde{P}_{B(x, s / 2)}(x, z) \tilde{u}(z) d z \\
& \approx \int_{B(y, s / 2)} P_{B(x, s / 2)}(x, z) \tilde{u}(z) d z \\
& \geq|B(y, s / 2)| C_{d, \alpha} 2^{-(d+2 \alpha)} 2^{-k(d+\alpha)} s^{-d} \tilde{u}(y)=c 2^{-k(d+\alpha)} \tilde{u}(y) .
\end{aligned}
$$

By symmetry, $\tilde{u}(x) \approx \tilde{u}(y)$.

We obtain a boundary Harnack principle for $L$ and general $C^{1,1}$ sets $D$.

Lemma 16 (BHP) Let $z \in \partial D, 0<r \leq r_{0}(D)$, and $0<p<1$. If $\tilde{u}$, $\tilde{v}$ are nonnegative in $\mathbb{R}^{d}$, regular L-harmonic in $D \cap B(z, r)$, vanish on $D^{c} \cap B(z, r)$ and satisfy $\tilde{u}\left(x_{0}\right)=$ $\tilde{v}\left(x_{0}\right)$ for some $x_{0} \in D \cap B(z, p r)$ then

$$
C^{-1} \tilde{v}(x) \leq \tilde{u}(x) \leq C \tilde{v}(x), \quad x \in D \cap B(z, p r),
$$

with $C=C\left(d, \alpha, b, p, r_{0}(D)\right)$.

Proof In view of Lemma 15 we may assume that $r$ is small. Let $F=F(z, r / 2) \subset$ $B(z, r)$ be the $C^{1,1}$ domain of Lemma 1 , localizing $D$ at $z$. For $x \in F$ we have $\tilde{u}(x)=\int \tilde{P}_{F}(x, z) \tilde{u}(z) d z \approx u(x)$, where $u(x)=\int P_{F}(x, z) \tilde{u}(z) d z$. Similarly $\tilde{v}(x) \approx$ $v(x)=\int P_{F}(x, z) \tilde{v}(z) d z$. Since $\tilde{u}\left(x_{0}\right)=\tilde{v}\left(x_{0}\right)$, we have $u\left(x_{0}\right) \approx v\left(x_{0}\right)$. By Lemma 3 , $u(x) \approx v(x)$, provided $x \in D \cap B(z, r / 8)$. We use Lemma 15 for the full range $x \in$ $D \cap B(z, p r)$.

\section{Proof of Theorem 1}

By Eqs. 52 and 29 we have the estimate

$$
\tilde{G}(x, y) \leq G(x, y)+d \int_{D} \frac{\tilde{G}(x, z) G(z, y)}{\delta(z) \wedge|y-z|}|b(z)| d z, \quad x, y \in D .
$$

We consider $\eta<1$, say $\eta=1 / 2$. By Lemma 9 and the uniform integrability in Lemma 11 (see Eq. 49) there is a constant $r>0$ so small that

$$
\int_{D^{r}} \frac{G(z, y)}{\delta(z) \wedge|y-z|}|b(z)| d z<\frac{\eta}{d}, \quad y \in D,
$$


and

$$
\int_{D^{r}} \frac{G(x, z) G(z, y)}{G(x, y)(\delta(z) \wedge|y-z|)}|b(z)| d z<\frac{\eta}{d}, \quad y \in D
$$

Here $D^{r}=\{z \in D: \delta(z) \leq r\}$. We denote

$$
\rho=\left[\varepsilon \wedge r_{0}(D) \wedge r\right] / 16
$$

with $\varepsilon=\varepsilon(d, \alpha, b, 2 / \kappa)$ of Lemma 13 , see also Lemma 1 .

To prove Eq. 1 we will consider $x, y$ in a partition of $D \times D$. We will also consider $Q, R \in \partial D$ such that $\delta(x)=|x-Q|, \delta(y)=|y-R|$.

I. First we suppose that $\delta(y) \geq \rho / 4$. We denote

- $D_{1}=\{x \in D:|y-x| \leq \rho / 8\}$,

- $D_{2}=\{x \in D: \delta(x) \geq \rho / 8\}$,

- $D_{3}=\{x \in D: \delta(x)<\rho / 8\}$.

(a) Let $x \in D_{1}$. Denote $B=B(y, \rho / 4)$. By Eq. 24 we have $G_{B}(x, y) \approx \mid x-$ $\left.y\right|^{\alpha-d} \approx G(x, y)$. Lemma 13 yields the lower bound in Eq. 1:

$$
\tilde{G}(x, y) \geq \tilde{G}_{B}(x, y) \approx G_{B}(x, y) \approx G(x, y) .
$$

By Lemma 7 we get the upper bound in Eq. 1.

(b) Let $x \in D_{2} \backslash D_{1}$. Let $x_{0} \in D$ be such that $\left|x_{0}-y\right|=\rho / 8$. $\tilde{G}(\cdot, y)$ is $L$ harmonic in $B(x, \rho / 8) \cup B\left(x_{0}, \rho / 8\right)$. By Lemma 15, (a) and Lemma 2 we get $\tilde{G}(x, y) \approx \tilde{G}\left(x_{0}, y\right) \approx G\left(x_{0}, y\right) \approx G(x, y)$.

(c) Let $x \in D_{3}$. Let $x_{0}, y_{0} \in D$ be collinear with $x, Q$ and such that $\delta\left(x_{0}\right)=$ $\left|x_{0}-Q\right|=5 \rho / 32$ and $\delta\left(y_{0}\right)=\left|y_{0}-Q\right|=7 \rho / 32$ (consider an inner ball tangent at $Q$ to see the situation). Let $F=F(Q, \rho)$ be the approximating domain of Lemma 1 . The functions $\tilde{G}\left(\cdot, y_{0}\right), \tilde{G}_{F}\left(\cdot, y_{0}\right)$ and $\tilde{G}(\cdot, y)$ are regular $L$-harmonic in $B(Q, 6 \rho / 32)$. By Lemmas 16,13 and 3,

$$
\frac{\tilde{G}(x, y)}{\tilde{G}\left(x_{0}, y\right)} \approx \frac{\tilde{G}_{F}\left(x, y_{0}\right)}{\tilde{G}_{F}\left(x_{0}, y_{0}\right)} \approx \frac{G_{F}\left(x, y_{0}\right)}{G_{F}\left(x_{0}, y_{0}\right)} \approx \frac{G(x, y)}{G\left(x_{0}, y\right)} .
$$

By (a) and (b), $\tilde{G}\left(x_{0}, y\right) \approx G\left(x_{0}, y\right)$ and we obtain Eq. 1 in the considered case I, that is for $\delta(y) \geq \rho / 4$ and all $x \in D$.

Before we proceed to the next case we recall that $\tilde{G}$ is non-symmetric.

II. Suppose that $\delta(y) \leq \rho / 4$. The proof of Eq. 1 follows in three steps.

Step 1. We will first prove that $\tilde{G}(x, y) \geq c G(x, y), x \in D$.

To this end we denote

- $F_{1}=\{x \in D:|x-R| \leq \rho\}$,

- $F_{2}=\{x \in D: \delta(x) \geq \rho / 4\}$,

- $F_{3}=\{x \in D: \delta(x)<\rho / 4\}$.

(d) Let $x \in F_{1}$. Consider $F=F(R, 8 \rho)$. Then $\delta(x)=\delta_{F}(x)$ and $\delta(y)=\delta_{F}(y)$. Consequently, $G(x, y) \approx G_{F}(x, y)$, see Eq. 24. As before we have

$$
\tilde{G}(x, y) \geq \tilde{G}_{F}(x, y) \approx G_{F}(x, y) \approx G(x, y) .
$$


(e) Let $x \in F_{2} \backslash F_{1}$. Let $x_{0} \in D$ be collinear with $y, R$ and such that $\delta\left(x_{0}\right)=$ $\left|x_{0}-R\right|=\rho / 2$. We note that $|x-y| \geq 3 \rho / 4$ and $\left|x_{0}-y\right| \geq \rho / 4$. By Harnack inequalities and (d), $\tilde{G}(x, y) \approx \tilde{\tilde{G}}\left(x_{0}, y\right) \geq c G\left(x_{0}, y\right) \approx G(x, y)$.

(f) Let $x \in F_{3} \backslash F_{1}$. Let $z_{0} \in D$ be such that $\delta\left(z_{0}\right)=\left|z_{0}-R\right|=\rho / 3$ and let $x_{0} \in D$ be such that $\delta\left(x_{0}\right)=\left|x_{0}-Q\right|=\rho / 4$. We have $\left|x_{0}-y\right|>\rho / 2$ and $\left|z_{0}-y\right| \geq \rho / 12$. By Harnack inequalities and (d),

$$
\tilde{G}\left(x_{0}, y\right) \approx \tilde{G}\left(z_{0}, y\right) \geq c G\left(z_{0}, y\right) \approx G\left(x_{0}, y\right) .
$$

$\tilde{G}\left(\cdot, z_{0}\right)$ and $\tilde{G}(\cdot, y)$ are regular $L$-harmonic in $D \cap B(Q, \rho / 3)$ because $\left|z_{0}-Q\right|>5 \rho / 12$ and $|y-Q|>\rho / 2$. By Lemmas 16, part I and 3,

$$
\frac{\tilde{G}(x, y)}{\tilde{G}\left(x_{0}, y\right)} \approx \frac{\tilde{G}\left(x, z_{0}\right)}{\tilde{G}\left(x_{0}, z_{0}\right)} \approx \frac{G\left(x, z_{0}\right)}{G\left(x_{0}, z_{0}\right)} \approx \frac{G(x, y)}{G\left(x_{0}, y\right)} .
$$

By this and Eq. 67, $\tilde{G}(x, y) \geq c G(x, y)$.

Step 2. We next prove the upper bound in Eq. 1 for $\delta(x) \geq \rho / 4$. By part I,

$$
c_{1}^{-1} G(x, z) \leq \tilde{G}(x, z) \leq c_{1} G(x, z), \quad z \in D \backslash D^{r} .
$$

The constant $c_{1}$ and other constants in what follows will only depend on $d, \alpha$, (the suprema in the Kato condition for) $b, r_{0}(D)$ and $\operatorname{diam}(D)$. By Eq. 45 and Lemma 11,

$$
\int_{D} \frac{G(x, z) G(z, y)}{\delta(z) \wedge|y-z|}|b(z)| d z \leq C_{1} G(x, y) .
$$

Therefore by Eq. 64,

$$
\begin{aligned}
\tilde{G}(x, y) \leq & G(x, y)+c_{1} d \int_{D \backslash D^{r}} \frac{G(x, z) G(z, y)}{\delta(z) \wedge|y-z|}|b(z)| d z \\
& +d \int_{D^{r}} \frac{\tilde{G}(x, z) G(z, y)}{\delta(z) \wedge|y-z|}|b(z)| d z \\
\leq & A G(x, y)+d \int_{D^{r}} \frac{\tilde{G}(x, z) G(z, y)}{|y-z| \wedge \delta(z)}|b(z)| d z,
\end{aligned}
$$

where $A=1+c_{1} d C_{1}$. By Lemma 7 and Eq. 65 we obtain

$$
\begin{aligned}
\tilde{G}(x, y) & \leq A G(x, y)+C_{0} d \int_{D^{r}} \frac{|x-z|^{\alpha-d} G(z, y)}{\delta(z) \wedge|y-z|}|b(z)| d z \\
& \leq A G(x, y)+B(x),
\end{aligned}
$$

where $B(x)=\eta C_{0} \delta_{D^{r}}(x)^{\alpha-d}$. We claim that for $n=0,1, \ldots$,

$$
\tilde{G}(x, y) \leq A\left(1+\eta+\cdots+\eta^{n}\right) G(x, y)+\eta^{n} B(x) .
$$

This is proved by induction: we plug Eq. 70 into Eq. 68, and use Eqs. 65 and 66. In consequence,

$$
\tilde{G}(x, y) \leq \frac{A}{1-\eta} G(x, y) .
$$


Step 3. We will now prove the upper bound in Eq. 1 when $\delta(x)<\rho / 4$. We will consider $F_{1}, F_{3}$ and $F$ from Step 1. If $x \in F_{3} \backslash F_{1}$ than we use the same argument as in (f), but this time all the terms in Eq. 67 are comparable because of Eq. 71, and we obtain Eq. 1. Finally, for $x \in F_{1} \subset F$ we have

$$
\tilde{G}(x, y)=\tilde{G}_{F}(x, y)+\int_{D \backslash F} \tilde{P}_{F}(x, z) \tilde{G}(z, y) d z .
$$

By Lemma $13, \tilde{G}_{F}(x, y) \approx G_{F}(x, y)$. We already know that for $z \in$ $D \backslash F_{1}, \tilde{G}(z, y) \approx G(z, y)$, and $\tilde{P}_{F}(x, z) \approx P_{F}(x, z)$ by Eq. 60 . Thus,

$$
\tilde{G}(x, y) \approx G_{F}(x, y)+\int_{D \backslash F} P_{F}(x, z) G(z, y) d z=G(x, y) .
$$

The proof of Theorem 1 is complete. In passing we only note that Eqs. 38 and 1, and [20] or [30, Theorem 22] yield sharp estimates of the Poisson kernel:

$$
\tilde{P}_{D}(x, y) \approx P_{D}(x, y) \approx \delta_{D}(x)^{\alpha / 2} \delta_{D^{c}}(y)^{-\alpha / 2}\left[1 \vee \delta_{D^{c}}(y)\right]^{-\alpha / 2}|y-x|^{-d} .
$$

\section{Appendix}

Let function $\phi$ be of class $C^{1,1}$, i.e. satisfy

$$
|\nabla \phi(\tilde{x})-\nabla \phi(\tilde{y})| \leq \eta|\tilde{x}-\tilde{y}|, \quad \tilde{x}, \tilde{y} \in \mathbb{R}^{d-1},
$$

for some $\eta<\infty$. Let $f(t)=\phi((1-t) \tilde{y}+t \tilde{x}), t \in[0,1]$. We have

$$
\begin{aligned}
|\phi(\tilde{x})-\phi(\tilde{y})-\nabla \phi(\tilde{y}) \cdot(\tilde{x}-\tilde{y})| & =\left|\int_{0}^{1}\left(f^{\prime}(t)-f^{\prime}(0)\right) d t\right| \\
& =\left|\int_{0}^{1}(\tilde{x}-\tilde{y}) \cdot(\nabla \phi((1-t) \tilde{y}+t \tilde{x})-\nabla \phi(\tilde{y})) d t\right| \\
& \leq \eta|\tilde{x}-\tilde{y}|^{2} / 2
\end{aligned}
$$

We will consider nonlinear transformations of $\mathbb{R}^{d}$ defined as follows,

$$
T x=\left(\tilde{x}, x_{d}+\phi(\tilde{x})\right), \quad T^{-1} x=\left(\tilde{x}, x_{d}-\phi(\tilde{x})\right) .
$$

Proof of Lemma 1 For $x=\left(x_{1}, \ldots, x_{d-1}, x_{d}\right) \in \mathbb{R}^{d}$ we let $\tilde{x}=\left(x_{1}, \ldots, x_{d-1}\right)$, so that $x=\left(\tilde{x}, x_{d}\right)$. The halfspace $H=\left\{x \in \mathbb{R}^{d}: x_{d}>0\right\}$ is a $C^{1,1}$ domain at each scale $r>0$, and we can localize it at 0 by

$$
K=\left\{x \in \mathbb{R}^{d}: 0<x_{d}<r / 2,|\tilde{x}|<r / 4+\sqrt{(r / 4)^{2}-\left(x_{d}-r / 4\right)^{2}}\right\} .
$$

Put differently, $K$ is defined by the conditions: $|\tilde{x}|<r / 2$ and

$$
r / 4-\sqrt{(r / 4)^{2}-\left((|\tilde{x}|-r / 4)_{+}\right)^{2}}<x_{d}<r / 4+\sqrt{(r / 4)^{2}-\left((|\tilde{x}|-r / 4)_{+}\right)^{2}},
$$


where $a_{+}=\max (a, 0)$. We see that $K$ is $C^{1,1}$ at scale $r / 4$. We consider the inner and outer balls of radius $r / 4$ for $K$, tangent at $Q \in \partial K$. Let

$$
B=\left\{x \in \mathbb{R}^{d}: 2(\mathrm{n}, x-Q)>|x-Q|^{2}\right\}
$$

be either one of them. Here $\mathrm{n} \in \mathbb{R}^{d}$ and $|\mathrm{n}|=r / 4$.

Let $D$ be $C^{1,1}$ at a scale $r>0$. It is well known that up to isometry $D$ locally coincides with the image of the halfspace $H$ by a transformation $T$ of the form (75), see [1, Section 2]. Namely, by possibly changing coordinates, we may assume that $0 \in \partial D, \phi$ satisfies Eq. 73, $\phi(0)=0, \nabla \phi(0)=0$,

$$
|\phi(\tilde{x})| \leq \frac{|\tilde{x}|^{2}}{r}<\frac{r}{4}, \quad \text { provided } \quad|\tilde{x}|<r / 2,
$$

and

$$
\left\{x \in D:|\tilde{x}|<r / 2,\left|x_{d}\right|<r\right\}=\left\{x \in \mathbb{R}^{d}:|\tilde{x}|<r / 2, \phi(\tilde{x})<x_{d}<r\right\} .
$$

We also have $\eta \leq c / r$ in Eq. 73, where $c \geq 1$ is an absolute constant.

We define $F=T K \subset D$. We see that $F$ locally coincides with $D$, and $F \subset\{x$ : $\left.|\tilde{x}|<r / 2,-r / 4<x_{d}<3 r / 4\right\}$, hence $\operatorname{diam}(F)<2 r$. We claim that $F$ is $C^{1,1}$. The claim will follow from considering the image of $B$ by $T$. Let $\xi=\nabla \phi(Q)$. We note that $|\xi| \leq \eta|\tilde{Q}| \leq \eta r / 2 \leq c / 2$. We define

$$
x \mapsto S x=T(Q+x)-T Q=\left(\tilde{x}, x_{d}+[\phi(\tilde{Q}+\tilde{x})-\phi(\tilde{Q})]\right),
$$

$S^{-1} x=\left(\tilde{x}, x_{d}-[\phi(\tilde{Q}+\tilde{x})-\phi(\tilde{Q})]\right), L x=\left(\tilde{x}, x_{d}+\xi \cdot \tilde{x}\right), L^{-1} x=\left(\tilde{x}, x_{d}-\xi \cdot \tilde{x}\right) . \mathrm{We}$ will use $L^{-1}$ as a linear approximation of $S^{-1}$ at $x=0$. Let $L^{-1 *}$ be the transpose of $L^{-1}$. We have $|L x| \leq|x|+|\xi||x| \leq|x|(2+c) / 2$. The same is true of $L^{-1}$ and $L^{-1 *}$. We note that

$$
T B-T Q=S(B-Q)=\left\{x \in \mathbb{R}^{d}: 2\left(\mathrm{n}, S^{-1} x\right)>\left|S^{-1} x\right|^{2}\right\},
$$

and $x=0$ is on the boundary of the set. Consider the ball $B^{\prime}=\left\{x: 2 \kappa\left(L^{-1 *} \mathrm{n}, x\right)>\right.$ $\left.|x|^{2}\right\}$. Note that $T Q \in \partial\left(B^{\prime}+T Q\right)$. We will verify our claim on $F$ by proving that if $2 \kappa=c^{-1} \wedge(1+3 c / 4)^{-2}$, then $B^{\prime} \subset S(B-Q)$, or $B^{\prime}+T Q \subset T B$. To this end we note that

$$
2 \kappa\left(L^{-1 *} \mathrm{n}, x\right)>|x|^{2}
$$

implies that $|x|<2 \kappa\left|L^{-1 *} \mathrm{n}\right| \leq r / 2$. For such (small) $x$, by Eq. 74, we obtain

$$
\left|S^{-1} x\right| \leq|x|+|\xi||x|+\eta|x|^{2} / 2 \leq|x|(1+3 c / 4) \text {. }
$$

Similarly,

$$
\left|S^{-1} x-L^{-1} x\right|=|\phi(\tilde{Q}+\tilde{x})-\phi(\tilde{x})-\xi \cdot \tilde{x}| \leq \eta|\tilde{x}|^{2} / 2 .
$$

Now, Eq. 76 yields $2\left(\mathrm{n}, L^{-1} x\right)>|x|^{2} / \kappa$, hence

$$
\begin{aligned}
2\left(\mathrm{n}, S^{-1} x\right) & >2\left(\mathrm{n}, S^{-1} x\right)-2\left(\mathrm{n}, L^{-1} x\right)+\frac{1}{\kappa}|x|^{2} \geq \frac{1}{\kappa}|x|^{2}-2|\mathrm{n}| \eta|\tilde{x}|^{2} / 2 \\
& \geq \frac{1}{2 \kappa}|x|^{2} \geq(2 \kappa)^{-1}(1+3 c / 4)^{-2}\left|S^{-1} x\right|^{2} \geq\left|S^{-1} x\right|^{2} .
\end{aligned}
$$

The proof is complete. 
Proof of Lemma 5 By [34] for $s>0, x \in \mathbb{R}^{d}$ and $\phi \in C_{c}^{\infty}\left((0, \infty) \times \mathbb{R}^{d}\right)$,

$$
\int_{s}^{\infty} \int_{\mathbb{R}^{d}} \tilde{p}(u-s, x, z)\left(\partial_{u}+\Delta_{z}^{\alpha / 2}+b(z) \cdot \nabla_{z}\right) \phi(u, z) d z d u=-\phi(s, x) .
$$

We note that the above integral is absolutely convergent. Indeed,

$$
\left|\left(\partial_{u}+\Delta_{z}^{\alpha / 2}+b(z) \cdot \nabla_{z}\right) \phi(u, z)\right| \leq c(1+|b(z)|),
$$

and if $\phi(u, z)=0$ for $u>M$, then by Eq. 33 and the remark following Eq. 30,

$$
\begin{aligned}
& \int_{s}^{\infty} \int_{\mathbb{R}^{d}} \tilde{p}(u-s, x, z)\left|\left(\partial_{u}+\Delta^{\alpha / 2}+b(z) \cdot \nabla_{z}\right) \phi(u, z)\right| d z d u \\
& \quad \leq c_{1} \int_{s}^{M} \int_{\mathbb{R}^{d}} p(u-s, x, z)(1+|b(z)|) d z d u \\
& \quad \leq c_{2} \int_{\mathbb{R}^{d}}\left(|x-z|^{\alpha-d} \wedge|x-z|^{-\alpha-d}\right)(1+|b(z)|) d z<\infty
\end{aligned}
$$

Furthermore, for $x \in \mathbb{R}^{d}, s \in \mathbb{R}$ by Eqs. 34 and 78 we obtain

$$
\begin{aligned}
\int_{s}^{\infty} & \int_{D} \tilde{p}_{D}(u-s, x, z)\left(\partial_{u}+\Delta_{z}^{\alpha / 2}+b(z) \cdot \nabla_{z}\right) \phi(u, z) d z d u \\
= & \int_{s}^{\infty} \int_{\mathbb{R}^{d}} \tilde{p}(u-s, x, z)\left(\partial_{u}+\Delta_{z}^{\alpha / 2}+b(z) \cdot \nabla_{z}\right) \phi(u, z) d z d u \\
& \quad-\tilde{\mathbb{E}}^{x} \int_{s+\tau_{D}}^{\infty} \int_{\mathbb{R}^{d}} \tilde{p}\left(u-s-\tau_{D}, X_{\tau_{D}}, z\right)\left(\partial_{u}+\Delta_{z}^{\alpha / 2}+b(z) \cdot \nabla_{z}\right) \phi(u, z) d z d u \\
= & -\phi(s, x)+\tilde{\mathbb{E}}^{x} \phi\left(s+\tau_{D}, X_{\tau_{D}}\right) \\
= & -\phi(s, x) .
\end{aligned}
$$

Proof of Lemma 6 Let $s=0, x \in D, \phi \in C_{c}^{\infty}\left((0, \infty) \times \mathbb{R}^{d}\right)$ and assume that supp $\phi \in$ $(0, \infty) \times(\bar{D})^{c}$. By Eqs. 79 and 5 we obtain

$$
\tilde{\mathbb{E}}^{x} \phi\left(\tau_{D}, X_{\tau_{D}}\right)=\int_{0}^{\infty} \int_{D} \int_{D^{c}} \tilde{p}_{D}(u, x, y) \phi(u, z) v(z-y) d z d y d u .
$$

Proof of Lemma 8 Let $\varphi \in C_{c}^{\infty}(D)$ and let $\chi(u) \in C_{c}^{\infty}(\mathbb{R})$ be such that $\chi(u)=1$ for $u \in(-1,1)$. For $n=1,2, \ldots$ we define $\phi_{n}(u, z)=\varphi(x) \chi(u / n)$, and we have

$$
\left|\left(\partial_{u}+\Delta^{\alpha / 2}+b(z) \cdot \nabla_{z}\right) \phi_{n}(u, z)\right| \leq c(1+|b(z)|)
$$


with $c$ independent of $n$. By Eq. 36, Lemma 7 and dominated convergence,

$$
\begin{aligned}
-\varphi(x) & =\lim _{n \rightarrow \infty}-\phi_{n}(0, x) \\
& =\lim _{n \rightarrow \infty} \int_{0}^{\infty} \int_{D} \tilde{p}_{D}(u, x, z)\left(\partial_{u}+\Delta_{z}^{\alpha / 2}+b(z) \cdot \nabla_{z}\right) \phi_{n}(u, z) d z d u \\
& =\int_{s}^{\infty} \int_{D} \tilde{p}_{D}(u, x, z)\left(\Delta^{\alpha / 2} \varphi(z)+b(z) \cdot \nabla \varphi(z)\right) d z d u \\
& =\int_{D} \tilde{G}_{D}(x, z)\left(\Delta^{\alpha / 2} \varphi(z)+b(z) \cdot \nabla \varphi(z)\right) d z .
\end{aligned}
$$

Acknowledgements The results were presented at the conference Nonlocal Operators and Partial Differential Equations, June 27-July 2, Bȩdlewo and The Sixth International Conference on Lévy Processes: Theory and Applications, July 26-30, 2010, Dresden. We thank the organizers for the invitation. In Dresden Professor Renming Song announced related sharp estimates of heat kernel $\tilde{p}_{D}$ for bounded $C^{1,1}$ open sets, analogous to [18].

Added in Proof The above mentioned results are available on arXiv [17].

Open Access This article is distributed under the terms of the Creative Commons Attribution Noncommercial License which permits any noncommercial use, distribution, and reproduction in any medium, provided the original author(s) and source are credited.

\section{References}

1. Aikawa, H., Kilpeläinen, T., Shanmugalingam, N., Zhong, X.: Boundary Harnack principle for $p$-harmonic functions in smooth Euclidean domains. Potential Anal. 26(3), 281-301 (2007)

2. Bliedtner, J., Hansen, W.: Potential Theory. Universitext. Springer, Berlin (1986). An analytic and probabilistic approach to balayage

3. Blumenthal, R.M., Getoor, R.K.: Markov processes and potential theory. In: Pure and Applied Mathematics, vol. 29. Academic, New York (1968)

4. Blumenthal, R.M., Getoor, R.K., Ray, D.B.: On the distribution of first hits for the symmetric stable processes. Trans. Am. Math. Soc. 99, 540-554 (1961)

5. Bogdan, K.: The boundary Harnack principle for the fractional Laplacian. Stud. Math. 123(1), 43-80 (1997)

6. Bogdan, K., Burdzy, K., Chen, Z.-Q.: Censored stable processes. Probab. Theory Relat. Fields 127(1), 89-152 (2003)

7. Bogdan, K., Byczkowski, T.: Potential theory for the $\alpha$-stable Schrödinger operator on bounded Lipschitz domains. Stud. Math. 133(1), 53-92 (1999)

8. Bogdan, K., Byczkowski, T.: Potential theory of Schrödinger operator based on fractional Laplacian. Probab. Math. Stat. 20(2, Acta Univ. Wratislav. No. 2256), 293-335 (2000)

9. Bogdan, K., Byczkowski, T., Kulczycki, T., Ryznar, M., Song, R., Vondraček, Z.: Potential analysis of stable processes and its extensions. In: Graczyk, P., Stos, A. (eds.) Lecture Notes in Mathematics, vol. 1980. Springer, Berlin (2009)

10. Bogdan, K., Grzywny, T., Ryznar, M.: Heat kernel estimates for the fractional Laplacian with Dirichlet conditions. Ann. Probab. 38(5), 1901-1923 (2010)

11. Bogdan, K., Hansen, W., Jakubowski, T.: Time-dependent Schrödinger perturbations of transition densities. Stud. Math. 189(3), 235-254 (2008)

12. Bogdan, K., Jakubowski, T.: Estimates of heat kernel of fractional Laplacian perturbed by gradient operators. Commun. Math. Phys. 271(1), 179-198 (2007)

13. Bogdan, K., Kulczycki, T., Kwaśnicki, M.: Estimates and structure of $\alpha$-harmonic functions. Probab. Theory Relat. Fields 140(3-4), 345-381 (2008) 
14. Bogdan, K., Kulczycki, T., Nowak, A.: Gradient estimates for harmonic and $q$-harmonic functions of symmetric stable processes. Ill. J. Math. 46(2), 541-556 (2002)

15. Bogdan, K., Sztonyk, P.: Estimates of the potential kernel and Harnack's inequality for the anisotropic fractional Laplacian. Stud. Math. 181(2), 101-123 (2007)

16. Caffarelli, L.A., Vasseur, A.: Drift diffusion equations with fractional diffusion and the quasigeostrophic equation. Ann. Math. (2) 171(3), 1903-1930 (2010)

17. Chen, Z., Kim, P., Song, R.: Dirichlet heat kernel estimates for fractional Laplacian with gradient perturbation. To appear in Ann. Probab. http://www.math.uiuc.edu/ rsong/hkedflpgo.pdf (2010). Accessed 3 June 2011

18. Chen, Z.-Q., Kim, P., Song, R.: Heat kernel estimates for Dirichlet fractional Laplacian. J. Eur. Math. Soc. 12(5), 1307-1329 (2010)

19. Chen, Z.-Q., Song, R.: Intrinsic ultracontractivity and conditional gauge for symmetric stable processes. J. Funct. Anal. 150(1), 204-239 (1997)

20. Chen, Z.-Q., Song, R.: Estimates on Green functions and Poisson kernels for symmetric stable processes. Math. Ann. 312(3), 465-501 (1998)

21. Chen, Z.-Q., Song, R.: General gauge and conditional gauge theorems. Ann. Probab. 30(3), 13131339 (2002)

22. Chung, K.L., Zhao, Z.X.: From Brownian motion to Schrödinger's equation. In: Grundlehren der Mathematischen Wissenschaften [Fundamental Principles of Mathematical Sciences], vol. 312. Springer, Berlin (1995)

23. Cranston, M., Zhao, Z.: Conditional transformation of drift formula and potential theory for $\frac{1}{2} \Delta+b(\cdot) \cdot \nabla$. Commun. Math. Phys. 112(4), 613-625 (1987)

24. Grzywny, T., Ryznar, M.: Estimates of Green functions for some perturbations of fractional Laplacian. Ill. J. Math. 51(4), 1409-1438 (2007)

25. Hansen, W.: Uniform boundary Harnack principle and generalized triangle property. J. Funct. Anal. 226(2), 452-484 (2005)

26. Hansen, W.: Global comparison of perturbed Green functions. Math. Ann. 334(3), 643-678 (2006)

27. Hunt, G.A.: Some theorems concerning Brownian motion. Trans. Am. Math. Soc. 81, 294-319 (1956)

28. Ifra, A., Riahi, L.: Estimates of Green functions and harmonic measures for elliptic operators with singular drift terms. Publ. Mat. 49(1), 159-177 (2005)

29. Ikeda, N., Watanabe, S.: On some relations between the harmonic measure and the Lévy measure for a certain class of Markov processes. J. Math. Kyoto Univ. 2, 79-95 (1962)

30. Jakubowski, T.: The estimates for the Green function in Lipschitz domains for the symmetric stable processes. Probab. Math. Stat. 22(2, Acta Univ. Wratislav. No. 2470), 419-441 (2002)

31. Jakubowski, T.: The estimates of the mean first exit time from a ball for the $\alpha$-stable OrnsteinUhlenbeck processes. Stoch. Process. Their Appl. 117(10), 1540-1560 (2007)

32. Jakubowski, T.: On Harnack inequality for $\alpha$-stable Ornstein-Uhlenbeck processes. Math. Z. 258(3), 609-628 (2008)

33. Jakubowski, T.: On combinatorics of Schrödinger perturbations. Potential Anal. 31(1), 45-55 (2009)

34. Jakubowski, T., Szczypkowski, K.: Time-dependent gradient perturbations of fractional Laplacian. J. Evol. Equ. 10(2), 319-339 (2010)

35. Kim, P., Lee, Y.-R.: Generalized $3 \mathrm{G}$ theorem and application to relativistic stable process on non-smooth open sets. J. Funct. Anal. 246(1), 113-143 (2007)

36. Kim, P., Song, R.: Two-sided estimates on the density of Brownian motion with singular drift. Ill. J. Math. 50(1-4), 635-688 (electronic) (2006)

37. Kiselev, A., Nazarov, F., Volberg, A.: Global well-posedness for the critical 2D dissipative quasigeostrophic equation. Invent. Math. 167(3), 445-453 (2007)

38. Kulczycki, T.: Properties of Green function of symmetric stable processes. Probab. Math. Stat. 17(2, Acta Univ. Wratislav. No. 2029), 339-364 (1997)

39. Landkof, N.S.: Foundations of Modern Potential Theory. Springer, New York (1972). Translated from the Russian by A. P. Doohovskoy, Die Grundlehren der mathematischen Wissenschaften, Band 180

40. Liskevich, V., Zhang, Q.S.: Extra regularity for parabolic equations with drift terms. Manuscr. Math. 113(2), 191-209 (2004)

41. Riesz, M.: Intégrales de Riemann-Liouville et potentiels. Acta Sci. Math. (Szeged) 9, 1-42 (1938) 
42. Sato, K.-i.: Lévy processes and infinitely divisible distributions. In: Cambridge Studies in Advanced Mathematics, vol. 68. Cambridge University Press, Cambridge (1999). Translated from the 1990 Japanese original, Revised by the author

43. Schilling, R.L., Song, R., Vondraček, Z.: Bernstein functions. In: de Gruyter Studies in Mathematics, vol. 37. Walter de Gruyter \& Co., Berlin (2010) Theory and applications

44. Song, R.M.: Probabilistic approach to the Dirichlet problem of perturbed stable processes. Probab. Theory Relat. Fields 95(3), 371-389 (1993)

45. Song, R.M.: Feynman-Kac semigroup with discontinuous additive functionals. J. Theor. Probab. 8(4),727-762 (1995)

46. Zhang, Q.: A Harnack inequality for the equation $\nabla(a \nabla u)+b \nabla u=0$, when $|b| \in K_{n+1}$. Manuscr. Math. 89(1), 61-77 (1996)

47. Zhang, Q.S.: Gaussian bounds for the fundamental solutions of $\nabla(A \nabla u)+B \nabla u-u_{t}=0$. Manuscr. Math. 93(3), 381-390 (1997) 